\title{
Cohomology and Extensions of von Neumann Algebras. I
}

By

\author{
Colin E. SutherLAND*
}

\begin{abstract}
We study twisted crossed products of von Neumann algebras by locally compact groups of automorphisms, where the twist is measured by a two-cocycle on the group, with values in the unitary group in the centre of the algebra. After examining alternative definitions of the crossed product, we prove the existence of "dual weights" on the crossed product, and identify the modular objects associated with these dual weights. We give criteria under which an automorphism of the original algebra may be lifted to an automorphism of the crossed product, and prove conditions under which the twisted crossed product will coincide with an ordinary crossed product. We give examples; some arising from extension theory of abelian groups, and the others giving a construction of factors not anti-isomorphic with themselves.
\end{abstract}

\section{Introduction}

This paper is the first in a series concerned with extensions of von Neumann algebras by groups (locally compact) of automorphisms. Here we concentrate exclusively on the twisted crossed product of a von Neumann algebra $\mathscr{M}$ by a group $G$ of automorphisms, the twist arising from the introduction of a two-cocycle $\omega \in Z^{2}(G, \mathcal{U})$, where $\mathcal{U}$ is the centre of the unitary group of $\mathscr{M}$. This crossed product should be viewed as the von Neumann algebra analogue of the crossed product used in the study of central simple algebras, as well as the analogue of the theory of (topological) group extension. However the situation is more complicated in the von Neumann algebra setting than in either of the prototypes; the natural algebraic conditions on a von Neumann algebra $\mathscr{N}$ for it to be an extension of $\mathscr{M}$ by $G$ are not strong enough to ensure that $\mathscr{N}$ is a twisted crossed product of $\mathscr{M}$ by $G$. A systematic study of the

Communicated by H. Araki, December 6, 1977.

* Department of Mathematics, Queen's University, Kingston, Ontario, K7L 3N6, Canada. Current address: Department of Mathematics, University of Oregon, Eugene, Oregon 97403, U.S.A. 
extension theory of will be given in a subsequent paper; in particular necessary and sufficient conditions will be developed to characterize the twisted crossed products among all extensions.

The organization of the paper is as follows; $\mathscr{M}$ is a von Neumann algebra, $\left\{\alpha_{g}: g \in G\right\}$ a representation of the locally compact group $G$ in the automorphism group of $\mathcal{M}$, and $\omega \in Z_{a}^{2}(G, \mathcal{U})$ as above; for technical reasons we assume $\mathscr{M}_{*}$ and $G$ are both separable. In Section 2 we define, and develop elementary properties of the crossed product $\mathscr{R}(\mathscr{M}, G, \alpha, \omega)$; this definition is a straight forward generalization of the works of Nakamura and Takeda [16], Zeller-Meier [24], and Takesaki [23]. We also give an alternative definition, utilizing the ideas of Hilbert modules and induced representations as developed by Paschke [17] and Rieffel [20].

Next we show how to associate to each faithful normal semifinite weight $\phi$ on $\mathscr{M}$, a weight $\tilde{\phi}$ on $\mathscr{R}(\mathscr{M} ; G ; \alpha ; \omega)$ which is dual to $\phi$, the duality being expressed by the relation of the modular objects for $\phi$ to those of $\tilde{\phi}$, and a kind of "Plancherel property" for the weights $\phi$ and $\tilde{\phi}$. It will be seen in [21] that it is this Plancherel property that distinguishes the twisted crossed products. The results given here are fairly easy generalizations of those given in [21], and by Digernes in [7].

In Section 4 we examine which automorphisms of $\mathscr{M}$ admit liftings to $\mathscr{R}(\mathscr{M}, G, \alpha, \omega)$; this is shown to be equivalent to a problem in non-abelian cohomology (coefficients in the unitary group of $\mathscr{M}$ ), and an interpretation of $Z_{a}^{1}(G, \mathcal{U})$ is given as certain liftings of the identity automorphism of $\mathscr{M}$.

The final two sections are concerned with the following problem; when can a twisted crossed product $\mathscr{R}(\mathscr{M}, G, \alpha, \omega)$ be expressed as an ordinary crossed product $\mathscr{R}(\mathscr{M}, G, \beta)$ for some action $\beta$ of $G$ on $\mathscr{M}$ ? In Section 5 we see that this is always possible when $\mathscr{M}$ is a properly infinite factor as well as some other cases, while in Section 6 we see (by example) that this is not the case when $\mathscr{M}$ is abelian. In particular, in Section 6 we construct Connes examples $Q_{\lambda, p, \gamma}$, [3], of type III factors not anti-isomorphic with themselves as the twisted crossed product of an abelian algebra by discrete groups of automorphisms; we also demonstrate a precise analogy of the von Neumann algebra theory with the extension theory of locally compact (separable) abelian groups.

We should point out that this paper is intended to lay a very general base for the study of twisted crossed products; it is not intended to give detailed information on the many interesting special situations to which the theory may be applied. We hope to present such information at a later date. 


\section{§1. Preliminaries and Notations}

Throughout, $\mathscr{M}$ will denote a von Neumann algebra with separable predual $\mathscr{N}_{*}$, and $G$ a locally compact separable group; on $G$ we will invariably work with a left Haar measure; the modular function of $G$ will be denoted $\delta_{G}$. If $\mathcal{H}$ is a separable Hilbert space, $L^{2}(G ; \mathcal{H})$ will denote the Hilbert space of equivalence classes of measurable functions $\xi: G \rightarrow \mathcal{H}$ for which $\int_{G}\|\xi(g)\|^{2} d g<\infty$.

The centre of $\mathscr{M}$ will be denoted $\mathscr{L}(\mathscr{M})$, the unitary group of $\mathscr{M}$ is $\mathcal{U}(\mathscr{M})$, and the group of all automorphisms of $\mathscr{M}$ will be written $\operatorname{Aut}(\mathscr{M})$. When we refer to a Borel structure on $\mathcal{U}(\mathscr{M})$, we mean that Borel structure generated by the strong-*-topology on $\mathcal{U}(\mathscr{M})$ (this structure is standard); $\operatorname{Aut}(\mathscr{M})$ will be considered as a topological group, the topology being that of pointwise norm convergence on $\mathscr{M}_{*}$ (see [2], [9]).

If $G$ and $\mathscr{M}$ are given, a representation of $G$ on $\mathscr{M}$, or in $\operatorname{Aut}(\mathscr{M})$, means a homomorphism $\alpha: g \in G \rightarrow \alpha_{g} \in \operatorname{Aut}(\mathscr{M})$ which is continuous; in particular, for $x \in \mathscr{M}$, the map $g \in G \rightarrow \alpha_{g}(x)$ is strong $*$-continuous. If $\left\{\alpha_{g}: g \in G\right\}$ is a representation of $G$ on $\mathscr{M}$, then for each $g \in G, \alpha_{g}(\mathscr{Z}(\mathscr{M}))=\mathscr{Z}(\mathscr{M})$, and thus $G$ acts on the Polish abelian group $\mathcal{U}(\mathscr{Z}(\mathscr{M}))$; it is this action which gives rise to the cohomology groups which will be of interest.

Let $A$ denote a Polish abelian group, and $\left\{\alpha_{g}: g \in G\right\}$ be a group of homeomorphisms of $A$, with $(g, a) \in G \times A \rightarrow \alpha_{g}(\alpha)$ being continuous. In [15], C.C. Moore has defined cohomology groups $H_{a}^{n}(G ; A)$ appropriate to this situation. If $G$ is descrete (and hence countable) these reduce to the classical, algebraically defined cohomology groups (see [14]). If $G$ is not discrete they may be defined as follows; let $C^{n}(G, A)$ denote the space of all Borel functions $\phi: G \times G \times \cdots \times G$ ( $n$ copies) $\rightarrow A$; we refer to such functions as $n$-cochains. Define boundary maps $\partial_{n}: C^{n}(G, A) \rightarrow C^{n+1}(G, A)$ by the formula

$$
\begin{aligned}
& \left(\partial_{n} \phi\right)\left(g_{1}, \cdots, g_{n+1}\right)=\alpha_{g_{1}}\left(\phi\left(g_{2}, \cdots, g_{n+1}\right)\right) \\
+ & \sum_{j=1}^{n}(-1)^{j} \phi\left(g_{1}, \cdots, g_{j} g_{j+1}, \cdots, g_{n+1}\right)+(-1)^{n+1} \phi\left(g_{1}, \cdots, g_{n}\right) .
\end{aligned}
$$

(We write $A$ additively, and $G$ multiplicatively.)

It is trivially verified that $\partial_{n+1} \bullet \partial_{n}=0$ for all $n$; the cohomology groups $H_{\alpha}^{n}(G ; A)$ are defined as the quotients $H_{a}^{n}(G ; A)=Z_{a}^{n}(G ; A) / B_{a}^{n}(G ; A)$, where $Z_{a}^{n}(G ; A)$ $=\operatorname{kernel}\left(\partial_{n}\right)$ are the $n$-cocycles, and $B_{a}^{n}(G ; A)=\operatorname{Image}\left(\partial_{n-1}\right)$ are the boundaries. As usual we write $\partial$ for $\partial_{n}$. It should be remarked that Moore gives an alternative definition using equivalence classes of elements of $C^{n}(G ; A)$ as $n$ - 
cochains; this is preferable from a topological point of view, but will play little role here. For our purposes, it will be more convenient to have at our disposal cocycles and cochains $\phi$ satisfying the additional property that $\phi\left(g_{1}, \cdots\right.$, $\left.g_{n}\right)=0$ whenever any one of the variables $g_{1}, \cdots, g_{n}$ is the identity; such cochains will be said to be normalized. In $[8, \S 6]$ it is proved that any cohomology class (with arbitrary cochains) contains a normalized element, and that any two such elements differ by the boundary of a normalized cochain; the proof given there is for the purely algebraic case, but obviously works in the context of Borel maps.

\section{§2. Crossed Products with Cocycles; Elementary Properties}

Here our context will be as follows: $\mathscr{M}$ is a von Neumann algebra and $g \in G \rightarrow \alpha_{g} \in$ Aut $\mathscr{M}$ a continuous representation of $G$ on $\mathscr{M}$. We let $\omega \in$ $Z_{\alpha}^{2}(G, \mathcal{U}(\mathscr{Z}(\mathscr{H})))$ be a fixed normalized Borel two cocycle. We shall refer to the system $\{\mathscr{M}, G, \alpha, \omega\}$ as a projective covariant system (usually abbreviated p.c.s.).

We shall give two definitions of the crossed product of $\mathscr{M}$ by $\alpha$ and $\omega$; the first is an immediate generalization of the definitions of Zeller-Meier [24] and Takesaki [23]; the second is independent of any particular realization of $\mathscr{M}$ on Hilbert space, and uses the theory of inner product modules over von Neumann algebras, [17] and induced representations [20].

Definition 2.1 Let $\{\mathscr{M}, G, \alpha, \omega\}$ be a p.c.s., and suppose $\mathscr{M}$ acts on the Hilbert space $\mathscr{H}$. The crossed product of $\mathscr{M}$ by $\alpha$ and $\omega$, denoted $\mathscr{R}(\mathscr{M}, G$, $\alpha, \omega)$ is the von Neumann algebra on $L^{2}(G, \mathscr{H})$ generated by the operators

$$
\begin{cases}\left(I^{\omega}(x) \xi\right)(g)=\alpha_{g}^{-1}(x) \xi(g), & x \in \mathscr{M} \\ \left(\lambda^{\omega}(h) \xi\right)(g)=\omega\left(g^{-1}, h\right) \xi\left(h^{-1} g\right), & h \in G\end{cases}
$$

where $\xi \in L^{2}(G, \mathcal{H})$.

Remarks. 1) If $\omega(g, h)=1$ for all $g, h \in G$ (we write $\omega=\iota$ ) the above definition reduces to the crossed product defined by Takesaki in [23].

2) If $G$ is descrete, our definition is (a unitary transform of) that given by Zeller-Meier in [24].

3) If $\mathscr{M}$ is a factor, or if the values of $\omega$ are scalars, $\lambda^{\omega}$ is (the identity tensor) the left regular $\omega$-representation of $G$. Our theory will thus apply to the algebras generated by regular projective representations of locally 
pact groups.

Proposition 2.2. Let $\{\mathscr{M}, G, \alpha, \omega\}$ be a p.c.s. Then the generators $I^{\alpha}(x)$, $\lambda^{\omega}(h)$ of $\mathscr{R}(\mathcal{M}, G, \alpha, \omega)$ satisfy the relations

$$
\begin{array}{ll}
\operatorname{Ad} \lambda^{\omega}(h)\left(I^{\alpha}(x)\right)=I^{\alpha}\left(\alpha_{h}(x)\right), & x \in \mathscr{M}, h \in G ; \\
\lambda^{\omega}(g) \lambda^{\omega}(h)=I^{\alpha}(\omega(g, h)) \lambda^{\omega}(g h), & g, h \in G .
\end{array}
$$

Proof: We compute, with $\xi \in L^{2}(G, \mathcal{H})$,

$$
\begin{aligned}
\left(\lambda^{\omega}(h) I^{\omega}(x) \lambda^{\omega}(h)^{* \xi}\right)(g) & =\omega\left(g^{-1}, h\right) \alpha_{g^{-1} h}(x) \omega\left(g^{-1}, h\right)^{* \xi(g)} \\
& =\alpha_{g^{-1} h}(x) \xi(g) \\
& =\left(I^{\omega}\left(\alpha_{h}(x)\right) \xi\right)(g)
\end{aligned}
$$

and

$$
\begin{aligned}
\left(\lambda^{\omega}(g) \lambda^{\omega}(h) \xi\right)(k) & =\omega\left(k^{-1}, g\right) \omega\left(k^{-1} g, h\right) \xi\left(h^{-1} g^{-1} k\right) \\
& =\alpha_{k}^{-1}(\omega(g, h)) \omega\left(k^{-1}, g h\right) \xi\left(h^{-1} g^{-1} k\right) \\
& =\alpha_{k}^{-1}(\omega(g, h)) \lambda^{\omega}((g h) \xi)(k) \\
& =\left(I^{\omega}(\omega(g, h)) \lambda^{\omega}(g h) \xi\right)(k),
\end{aligned}
$$

where we have used the cocycle identity for $\omega$ and the fact that $\omega$ takes values in the centre of $\mathscr{M}$.

Q.E.D.

Our definition of the crossed product apparently depends on the Hilbert space on which $\mathscr{M}$ is realized. This is not the case; the proof of this fact is identical with that given by Takesaki in [23] for the case of ordinary crossed products. We record this as

Proposition 2.3. Let $\{\mathscr{M}, G, \alpha, \omega\}$ and $\{\mathscr{l}, G, \beta, \nu\}$ be p.c.s.'s. If there is a normal isomorphism $\kappa: \mathscr{M} \rightarrow \mathcal{I}$ with $\kappa \circ \alpha=\beta \circ \kappa$ and $\kappa \circ \omega=\nu \circ \kappa$, then there is a normal isomorphism $\vec{\kappa}: \mathscr{R}(\mathscr{M}, G, \alpha, \omega) \rightarrow \mathscr{R}(\mathcal{R}, G, \beta, \nu)$ with $\bar{\kappa}\left(I^{\alpha}(x)\right)$ $=I^{\beta}(\kappa(x)), x \in \mathscr{M}$, and $\bar{\kappa}\left(\lambda^{\omega}(g)\right)=\lambda^{\nu}(g), g \in G$.

The other ingredients of our crossed product, namely the action of $G$ and the cocycle may be changed within certain limits to produce isomorphic crossed products.

Proposition 2.4. Let $\{\mathscr{M}, G, \alpha, \omega\}$ and $\{\mathscr{M}, G, \beta, \nu\}$ be p.c.s.'s. Then if either

(i) $\alpha=\beta$ and $\omega$ is cohomologous to $\nu$

or 
(ii) $\omega=\nu$, and there is a Borel map $g \in G \rightarrow u_{g} \in \mathcal{U}(\mathscr{M})$ with $u_{g h}=u_{g} \alpha_{g}\left(u_{h}\right)$ and $\beta_{g}=\operatorname{Ad} u_{g} \circ \alpha_{g}(g, h \in G)$, then there is a normal isomorphism $\kappa: \mathscr{R}(\mathscr{M}, G, \alpha, \omega)$ $\rightarrow \mathscr{R}(\mathscr{M}, G, \beta, \nu)$ with $\kappa\left(I^{\omega}(x)\right)=I^{\beta}(x), x \in \mathscr{M}$ and $\kappa\left(\lambda^{\omega}(g)\right) \in I^{\beta}(\mathscr{M}) \lambda^{\nu}(g), g \in G$.

Proof. We may suppose that $\mathscr{M}$ acts on the Hilbert space $\mathscr{H}$ and that and crossed products involved act on $L^{2}(G, \mathcal{H})$.

(i) If $\omega$ is cohomologous to $\nu$ we may choose a Borel map $b: g \in G \rightarrow$ $b_{g} \in \mathcal{U}(\mathscr{Z}(\mathscr{M}))$ with $\omega(g, h)=\alpha_{g}\left(b_{h}\right) b_{g h}^{*} b_{g} \nu(g, h)$ for all $g, h \in G$. Define a unitary $B$ on $L^{2}(G, \mathcal{H})$ by $(B \xi)(g)=b_{g^{-1}}^{*} \xi(g), \xi \in L^{2}(G, \mathcal{H})$. Direct computation yields $B I^{\alpha}(x) B^{*}=I^{\alpha}(x)=I^{\beta}(x)$ and $B \lambda^{\omega}(h) B^{*}=I^{\alpha}\left(b_{h}\right) \lambda^{\nu}(h), x \in \mathscr{M}, h \in G$, and we may take $\bar{\kappa}=\operatorname{Ad} B$.

(ii) We first note that if $\beta_{g}=\operatorname{Ad} u_{g} \circ \alpha_{g}$ then $Z_{\alpha}^{2}(G, \mathcal{U}(\mathscr{Z}(\mathscr{M}))$ $=Z_{\beta}^{2}(G, \mathcal{U}(\mathscr{Z}(\mathscr{M})))$. Now if $\omega, \nu, \alpha, \beta$ are as in (ii), define a unitary $U$ on $L^{2}(G, \mathscr{H})$ by $(U \xi)(g)=u_{g^{-1}} \xi(g)$. Another easy computation yields $U I^{\infty}(x) U^{*}$ $=I^{\beta}(x)$ and $U \lambda^{\omega}(h) U^{*}=I^{\beta}\left(u_{h}^{*}\right) \lambda^{\omega}(h)=I^{\beta}\left(u_{h}^{*}\right) \lambda^{\omega}(h)$. We set $\kappa=\operatorname{Ad} U$. Q.E.D.

For later purposes it will be convenient to have an alternative presentation of the generators for the crossed product in case the action of $G$ on $\mathscr{M}$ is implemented (on the Hilbert space $\mathscr{H}$ ).

Lemma 2.5. Let $\{\mathscr{M}, G, \alpha, \omega\}$ be a p.c.s. with $\mathscr{M}$ acting on $\mathscr{H}$. Suppose there is a group $g \rightarrow u_{g}$ of unitaries on $\mathscr{H}$ with $\alpha_{g}=\operatorname{Ad} u_{g}$. Then the operators

$$
\begin{gathered}
\tilde{I}(x)=x \otimes 1, \quad x \in \mathscr{M} \\
\left(\tilde{\lambda}^{\omega}(h) \xi\right)(g)=u_{h} \alpha_{h^{-1} g}\left(\omega\left(g^{-1}, h\right)\right) \xi\left(h^{-1} g\right),
\end{gathered}
$$

on $L^{2}(G ; \mathcal{H})=\mathscr{H} \otimes L^{2}(G)$ generate an algebra isomorphic with $\mathscr{R}(\mathscr{H}, G, \alpha, \omega)$.

Proof. Define a unitary $U$ on $L^{2}(G, \mathscr{H})$ by $(U \xi)(g)=u_{g} \xi(g)$. It is trivial to compute that $U I^{\alpha}(x) U^{*}=\widetilde{I}(x)$ and $U \lambda^{\omega}(h) U^{*}=\tilde{\lambda}^{\omega}(h) . \quad$ Q.E.D.

Remark. If $\omega$ takes scalar values, $\tilde{\lambda}^{\omega}(h)$ is nothing but $u_{h} \otimes \pi^{\omega}(h)$, where $\pi^{\omega}$ is the left regular $\omega$-representation of $G$.

For the sake of completeness we will give an alternative definition of the crossed product, one which is independent of any particular realization of $\mathscr{M}$ Hilbert space. The construction, and the proof that it does indeed give an alternative definition, rely on the machinery of inner product modules over von Neumann algebras and induced representations as developed in [20] and [17]. We will assume some familiarity with these results and techniques. The construction is also intimately related to the "dual weight constructions" 
as carried out in [23] and [7]; this relationship will be investigated more closely in Section 3.

Let $\{\mathscr{M}, G, \alpha, \omega\}$ be a p.c.s. We begin by defining the "twisted convolution algebra of functions on $G$ with values in $\mathcal{M}$," in analogy with the twisted group algebra associated to a scalar-valued two-cocycle on $G$. The fact that our cocycles are Borel rather than continuous complicates matters slightly.

Definition 2.6. $\mathcal{K}_{0}(\mathscr{M}, G)$ is the space of norm-bounded Borel maps $x: g \in G \rightarrow x(g) \in \mathscr{M}$ with $\{g: x(g) \neq 0\}$ precompact. $\mathcal{K}(\mathscr{M}, G)$ will denote the linear space of equivalence classes of elements of $\mathcal{K}_{0}(\mathscr{M}, G)$ under the equivalence $x \sim y$ if $x(g)=y(g)$ almost everywhere with respect to Haar measure on $G$.

We endow $\mathcal{K}_{0}(\mathscr{M}, G)$ with a product, an involution and an $\mathscr{M}$-valued pre-inner product as follows

Definition 2.7. For $x_{0}, y_{0} \in \mathcal{K}_{0}(\mathscr{M}, G)$ define

$$
\begin{gathered}
\left(x_{0} y_{0}\right)(g)=\int_{G} \alpha_{g}^{-1}\left(\omega\left(g h, h^{-1}\right)\right) \alpha_{h}\left(x_{0}(g h)\right) y_{0}\left(h^{-1}\right) d h, \\
x_{0}^{\sharp}(g)=\alpha_{g}^{-1}\left(x\left(g^{-1}\right)^{*}\right) \omega\left(g^{-1}, g\right)^{*} \delta_{G}\left(g^{-1}\right)
\end{gathered}
$$

and

$$
\left\langle x_{0}, y_{0}\right\rangle_{\mathscr{M}}=\int_{G} y_{0}(h)^{*} x_{0}(h) d h
$$

where $\delta_{G}$ is the modular function of $G$.

Remark. The above formula are motivated by thinking of $x_{0}$ as being represented in $\mathscr{R}(\mathscr{M}, G, \alpha, \omega)$ by the operator $\int_{G} \lambda^{\omega}(g) I^{\omega}\left(x_{0}(g)\right) d g$; while the identification of $x_{0}$ with $\int_{G} I^{\alpha}\left(x_{0}(g)\right) \lambda^{\omega}(g) d g$ gives rise to an equally valid algebraic structure on $\mathcal{K}_{0}(\mathscr{M}, G)$, various formulae (such as that for generators of the commutant of the crossed product) become more simple with the structure we have defined (see Section 3).

Lemma 2.8. With the product and involution as in $2.7, \mathcal{K}_{0}(\mathscr{M}, G)$ is an associative involutive algebra. Further, for $x_{0}, y_{0} \in \mathcal{K}_{0}(\mathscr{M}, G)$ the classes of $x_{0} y_{0}$ and $x_{0}^{\#}($ in $\mathcal{K}(\mathscr{M}, G))$ depend only on the classes of $x_{0}, y_{0}$ and $x_{0}$ respectively. Finally $\left\langle x_{0}, y_{0}\right\rangle_{\mathscr{M}}$ depends only on the classes of $x_{0}, y_{0}$ in $\mathcal{K}(\mathscr{M}, G)$.

Proof. For $x_{0} \in \mathcal{K}_{0}(\mathscr{M}, G)$ it is clear that $x_{0}^{\#} \in \mathcal{K}_{0}(\mathscr{M}, G)$ and that the 
class of $x_{0}^{\#}$ depends only on that of $x_{0}$. Also

$$
\begin{aligned}
x_{0}^{\#}(g) & =\alpha_{g}^{-1}\left(x_{0}^{\#}\left(g^{-1}\right)^{*}\right) \omega\left(g^{-1}, g\right)^{*} \delta_{G}\left(g^{-1}\right) \\
& =\alpha_{g}^{-1}\left(\alpha_{g}\left(x_{0}(g)\right)\right) \alpha_{g}^{-1}\left(\omega\left(g, g^{-1}\right)\right) \omega\left(g^{-1}, g\right) \\
& =x_{0}(g) .
\end{aligned}
$$

To check that the product lies in $\mathcal{K}_{0}(\mathscr{M}, G)$, let $x_{0}, y_{0} \in \mathcal{K}_{0}(\mathscr{H}, G)$ and $\psi \in \mathscr{M}_{*}^{+}$. Then

$$
\begin{aligned}
& \left|\int_{G} \psi\left(\alpha_{g}^{-1}\left(\omega\left(g h, h^{-1}\right)\right) \alpha_{h}\left(x_{0}(g h)\right) y_{0}\left(h^{-1}\right)\right) d h\right| \\
\leq & \int_{G}\left(\psi\left(\alpha_{h}\left(x_{0}(g h) x_{0}(g h)^{*}\right)\right)\right)^{1 / 2}\left(\psi\left(y_{0}\left(h^{-1}\right)^{*} y_{0}\left(h^{-1}\right)\right)\right)^{1 / 2} d h \\
\leq & \|\psi\| \int_{G}\left\|x_{0}(g h)\right\|\left\|y\left(h^{-1}\right)\right\| d h .
\end{aligned}
$$

Since $k \rightarrow\left\|x_{0}(k)\right\|,\left\|y_{0}(k)\right\|$ are bounded, Borel, and of compact support, $\left(x_{0} y_{0}\right)(g) \in \mathscr{M}$ for each $g$, and $g \rightarrow\left(x_{0} y_{0}\right)(g)$ is norm-bounded and of compact support; the map is also evidently Borel. Again it is clear that the class of $x_{0} y_{0}$ depends only on the classes of $x_{0}$ and $y_{0}$ in $\mathcal{K}_{0}(\mathscr{H}, G)$.

We check (formally) the necessary algebraic properties. For $x_{0}, y_{0}, z_{0} \in$ $\mathcal{K}_{0}(\mathscr{H}, G)$ we have (noting that by the cocycle identity $\alpha_{g^{-1}}\left(\omega\left(g h k, k^{-1} h^{-1}\right)\right)$. $\alpha_{h k}\left(\omega\left(k^{-1}, h^{-1}\right)\right)=\alpha_{g}^{-1}\left(\omega\left(g h, h^{-1}\right)\right) . \quad \alpha_{g}^{-1}\left(\omega\left(g h k, k^{-1}\right)\right)$ for all $\left.g, h, k \in G\right)$,

$$
\begin{aligned}
& \left(x_{0}\left(y_{0} z_{0}\right)\right)(g)=\int_{G} \alpha_{g}^{-1}\left(\omega\left(g l, l^{-1}\right)\right) \alpha_{l}\left(x_{0}(g l)\right)\left(y_{0} z_{0}\right)\left(l^{-1}\right) d l \\
& =\int_{G} \int_{G} \alpha_{g}^{-1}\left(\omega\left(g l, l^{-1}\right)\right) \alpha_{l}\left(\left(l^{-1} h, h^{-1}\right)\right) \alpha_{l}\left(x_{0}(g l)\right) \alpha_{h}\left(y_{0}\left(l^{-1} h\right)\right) Z_{0}\left(h^{-1}\right) d h d l \\
& =\int_{G} \int_{G} \alpha_{g}^{-1}\left(\omega\left(g h k, k^{-1} h^{-1}\right)\right) \alpha_{h k}\left(\omega\left(k^{-1}, h^{-1}\right)\right) \alpha_{h k}\left(x_{0}(g h k)\right) \alpha_{h}\left(y_{0}\left(k^{-1}\right)\right) z_{0}\left(h^{-1}\right) d k d h \\
& =\int_{G} \int_{G} \alpha_{g}^{-1}\left(\omega\left(g h, h^{-1}\right)\right) \alpha_{h}\left[\alpha_{g h}^{-1}\left(\omega\left(g h k, k^{-1}\right)\right) \alpha_{k}\left(x_{0}(g h k) y_{0}\left(k^{-1}\right)\right)\right] z_{0}\left(h^{-1}\right) d h d k \\
& =\int_{G} \alpha_{g}^{-1}\left(\omega\left(g h, h^{-1}\right)\right) \alpha_{h}\left(\left(x_{0} y_{0}\right)(g h)\right) z_{0}\left(h^{-1}\right) d h \\
& =\left(\left(x_{0} y_{0}\right) z_{0}\right)(g) .
\end{aligned}
$$

Also, if $x_{0}, y_{0} \in \mathcal{K}_{0}(\mathscr{M}, G)$, then

$$
\begin{aligned}
&\left(y_{0}^{\sharp} x_{0}^{\#}\right)(g) \\
&= \int_{G} \alpha_{g}^{-1}\left(\omega\left(g h, h^{-1}\right)\right) \alpha_{h}\left[\alpha_{g h}^{-1}\left(y_{0}\left(h^{-1} g^{-1}\right)^{*}\right) \omega\left(h^{-1} g^{-1}, g h\right)^{*}\right] \alpha_{h}\left(x_{0}(h)^{*}\right) \\
& \omega\left(h, h^{-1}\right)^{*} \delta_{G}(g)^{-1} d h
\end{aligned}
$$




$$
\begin{aligned}
& =\delta_{G}(g)^{-1} \int_{G} \alpha_{g}^{-1}\left(\omega\left(k, k^{-1} g\right)\right) \alpha_{g^{-1}}\left(\omega\left(k^{-1}, k\right)^{*}\right) \omega\left(g^{-1} k, k^{-1} g\right)^{*} \\
& \qquad \alpha_{g}^{-1}\left(y_{0}\left(k^{-1}\right)^{*}\right) \alpha_{g^{-1}}\left(x_{0}\left(g^{-1} k\right)^{*}\right) d k \\
& =\delta_{G}(g)^{-1} \omega\left(g^{-1}, g\right)^{*} \omega\left(g^{-1} k, k^{-1}\right)^{*} \alpha_{g}^{-1}\left(y_{0}\left(k^{-1}\right)^{*}\right) \alpha_{g^{-1}}\left(x_{0}\left(g^{-1} k\right)^{*}\right) d k \\
& =\delta_{G}(g)^{-1} \omega\left(g^{-1}, g\right)^{*} \alpha_{g}^{-1}\left(x_{0} y_{0}\right)\left(g^{-1}\right)^{*} \\
& =\left(x_{0} y_{0}\right)^{\#}(g),
\end{aligned}
$$

where we have used the identity

$$
\alpha_{g}^{-1}\left(\omega\left(k, k^{-1} g\right)\right) \alpha_{g^{-1} k}\left(\omega\left(k^{-1}, k\right)^{*}\right) \omega\left(g^{-1} k, k^{-1} g\right)^{*}=\omega\left(g^{-1}, g\right)^{*} \omega\left(g^{-1} k, k^{-1}\right),
$$

a consequence of the cocycle equation for $\omega$.

The proof that $\left\langle x_{0}, y_{0}\right\rangle \mathscr{M}_{\mathcal{M}}$ defines an element of $\mathscr{M}$ and that this element depends only on the classes of $x_{0}$ and $y_{0}$ in $\mathcal{K}(\mathscr{H}, G)$ is almost trivial, and is left to the reader.

Q.E.D.

From this point on, we shall largely ignore the distinction between $\mathcal{K}_{0}(\mathscr{M}, G)$, and $\mathcal{K}(\mathscr{M}, G)$; thus we assume that the product, involution and inner product are defined on $\mathcal{K}(\mathscr{M}, G)$.

Lemma 2.9. The inner product $\langle\cdot, \cdot\rangle_{\mathscr{M}}$ on $\mathcal{K}(\mathscr{M}, G)$ satisfies

i) $\langle x, x\rangle_{\mathscr{M}} \geq 0\left(\right.$ in $\mathscr{M}$ ) and $\langle x, x\rangle_{\mathscr{M}}=0$ only if $x=0$,

ii) $\langle x, y\rangle_{\mathscr{M}}=\langle y, x\rangle_{\mathscr{M}}^{*}$,

iii) $\left\{\langle x, y\rangle_{\mathscr{M}}: x, y \in \mathcal{K}(\mathscr{M}, G)\right\}=\mathscr{M}$.

Proof. i) That $\langle x, x\rangle_{\mathscr{M}} \geq 0$ in $\mathscr{M}$ is clear. If $x \in \mathcal{K}(\mathscr{M}, G),\langle x, x\rangle_{\mathscr{M}}$ $=0$ and $\psi \in \mathscr{M}_{*}$, then $0=\int_{G} \psi\left(x(g)^{*} x(g)\right) d g$, so that $\psi\left(x(g)^{*} x(g)\right)=0$ a.e. Letting $\psi$ run through a countable dense set in $\mathscr{M}_{*}^{+}$we see $x(g)=0$ a.e., and so $x=0$ in $\mathscr{K}(\mathscr{M}, G)$.

ii) trivial.

iii) if $z \in \mathscr{M}$, and $\Phi$ is a continuous compactly supported function on $G$ with $\int_{G}|\Phi(g)|^{2} d g=1$, then with $x(g)=\Phi(g) z$ and $y(g)=\Phi(g) 1$, we see $\langle x, y\rangle_{\mathscr{M}}=\int_{G}|\Phi(g)|^{2} z d g=z$, and $x, y \in \mathcal{K}(\mathscr{M}, G)$.

Q.E.D.

At this point, $\mathcal{K}(\mathscr{M}, G)$ is established as a right rigged space in the terminology of [20]. We give some other properties of $\mathcal{K}(\mathcal{M}, G)$, which are reminiscent of left Hilert algbebras.

Lemma 2.10. For $x, y, z \in \mathcal{K}(\mathcal{M}, G)$, we have 
i) $\langle x y, z\rangle_{\mathscr{M}}=\left\langle y, x^{*} z\right\rangle_{\mathscr{M}}$

ii) $\langle x y, x y\rangle_{\mathscr{M}} \leq\left\|\pi_{l}(\|x(\cdot)\|)\right\|^{2}\langle y, y\rangle_{\mathscr{M}^{\prime}}$, where $\pi_{l}(\|x(\cdot)\|)$ is the (bounded) operator of convolution on the left by the function $g \rightarrow\|x(g)\|$ on $L^{2}(G)$.

Proof. i) For $x, y, z \in \mathcal{K}(\mathscr{M}, G)$, we compute

$$
\begin{aligned}
\left\langle y, x^{\sharp} z\right\rangle_{\mathscr{M}} & =\int_{G} \int_{G} \alpha_{k}^{-1}\left(\omega\left(k l, l^{-1}\right)^{*}\right) \alpha_{l}\left[\alpha_{k l}^{-1}\left(x\left(l^{-1} k^{-1}\right)\right)^{*} \omega\left(l^{-1} k^{-1}, k l\right)^{*}\right. \\
& \left.\cdot \delta_{G}(k l)^{-1}\right] z\left(l^{-1}\right)^{*} y(k) d l d k \\
& =\int_{G} \int_{G} \alpha_{k}\left(\omega\left(k^{-1} l^{-1}, l\right)^{*}\right) \alpha_{l}^{-1}\left(\omega\left(l k, k^{-1} l^{-1}\right)\right) z(l)^{*} \\
& \cdot \alpha_{k}(x(l k)) y\left(k^{-1}\right) d l d k \\
& =\int_{G} \int_{G} \alpha_{l}^{-1}\left(\omega\left(l k, k^{-1}\right)\right) z(l)^{*} \alpha_{k}(x(l k)) y\left(k^{-1}\right) d l d k \\
& =\int_{G} z(l)^{*}(x y)(l) d l=\langle x y, z\rangle_{\mathscr{M}},
\end{aligned}
$$

where we have used the cocycle identity to replace $\alpha_{k}\left(\omega\left(k^{-1} l^{-1}, l\right)\right)^{*} . \quad \alpha_{l}^{-1}(\omega(l k$, $\left.\left.k^{-1} l^{-1}\right)\right)$ by $\alpha_{l}^{-1}\left(\omega\left(l k, k^{-1}\right)\right)$.

ii) For $x, y \in \mathcal{K}(\mathscr{M}, G)$ we compute, with $\psi \in \mathscr{M}_{*}^{+}$,

$$
\begin{aligned}
(x y)(g) & =\int_{G} \alpha_{g}^{-1}\left(\omega\left(k, k^{-1} g\right)\right) \alpha_{k^{-1} g}(x(k)) y\left(k^{-1} g\right) d k, \text { so } \\
\psi(\langle x y, x y)\rangle_{\mathscr{M}} & \leq \int_{G} \int_{G} \int_{G} \mid \psi\left(y ( k ^ { - 1 } g ) ^ { * } \alpha _ { k ^ { - 2 } g } ( x ( k ) ^ { * } ) \alpha _ { g } ^ { - 1 } \left(\omega\left(k, k^{-1} g\right)^{*}\right.\right. \\
& \left.\left.\leq \omega\left(h, h^{-1} g\right)\right) \alpha_{h^{-1} g}(y(h)) y\left(h^{-1} g\right)\right) \mid d h d k d g \\
& \leq \int_{G}\left(\int_{G} \psi\left(y\left(k^{-1} g\right)^{*} \alpha_{k^{-1} g}\left(x(k)^{*} x(k)\right) y\left(k^{-1} g\right)\right)^{1 / 2} d k\right)^{2} d g \\
& \leq \int_{G}^{m}\left(\int_{G}\|x(k)\| \psi\left(y\left(k^{-1} g\right)^{*} y\left(k^{-1} g\right)\right)^{1 / 2} d k\right)^{2} d g .
\end{aligned}
$$

With $Y_{\psi}(h)=\psi\left(y(h)^{*} y(h)\right)$ we see

$$
\begin{aligned}
\psi\left(\langle x y, x y\rangle_{\mathscr{M}}\right) & \leq\left\|\left(\|x(\cdot)\|^{*} Y_{\psi}\right)\right\|_{2}^{2} \\
& \leq \| \pi_{l}\left(x(\|\cdot\|)\left\|^{2}\right\| Y_{\psi} \|_{2}^{2}\right. \\
& =\left\|\pi_{l}(\|x(\cdot)\|)\right\|^{2} \int_{G} \psi\left(y(h)^{*} y(h)\right) d h \\
& =\left\|\pi_{l}(\|x(\cdot)\|)\right\|^{2} \psi\left(\langle y, y\rangle_{\mathscr{M}}\right) .
\end{aligned}
$$

Since $\psi$ is arbitrary, the proof is complete.

Q.E.D.

We now introduce on $\mathcal{K}(\mathscr{M}, G)$ the structure of a right $\mathscr{H}$-module-it is 
in fact also a left $\mathcal{K}(\mathscr{M}, G)$ module, and also a left $\mathscr{M}$-module, but for our purposes these structures are irrelevant.

Definition 2.11. For $x \in \mathcal{K}(\mathscr{M}, G), a \in \mathscr{H}$, define

$$
(x a)(g)=x(g) a .
$$

Lemma 2.12. i) With the above structure $\mathcal{K}(\mathscr{M}, G)$ is a right $\mathscr{M}$-module. ii) $\langle x a, y\rangle_{\mathscr{M}}=\langle x, y\rangle_{\mathscr{M}} a, x, y \in \mathcal{K}(\mathscr{M}, G), a \in \mathscr{M}$.

Proof. We leave the routine calculations to the reader.

Q.E.D.

Remark. In the terminology of [17], $\mathcal{K}(\mathscr{M}, G)$ is a prehilbert $\mathscr{M}$-module (Lemma 2.9 (i), (ii), 2.12 (i), (ii)); in the terminology [20] it is a left prehermitian $\mathscr{M}$-rigged $\boldsymbol{C}$-module (Lemmas 2.9, 2.12).

In order to apply the ideas of [17] and [20], we need to make one modification in our setup. Let $\mathscr{X}(\mathscr{M}, G)$ denote the self-dual completion of $\mathcal{K}(\mathscr{M}, G)$ (see [17]) and $\iota: \mathcal{K}(\mathscr{M}, G) \rightarrow \mathscr{K}(\mathscr{M}, G)$ be the canonical inclusion (denoted $x \rightarrow \tilde{x}$ in [17]). Since bounded module maps of $\mathcal{K}(\mathscr{M}, G)$ extend uniquely to $\mathscr{X}(\mathscr{M}, G)$, we have, by Lemma 2.10 (ii) an action of $\mathcal{H}(\mathscr{M}, G)$ on $\mathscr{X}(\mathscr{M}, G)$ as bounded $\mathscr{H}$-module maps $\left\{\pi_{l}(x): x \in \mathcal{K}(\mathscr{M}, G)\right\}$ determined by $\pi_{l}(x) \iota(y)$ $=\imath(x y)(x, y \in \mathcal{K}(\mathscr{M}, G))$. By [17], $\mathscr{X}(\mathscr{M}, G)$ is a (comglete) Hilbert $\mathscr{M}$-module so that the algebra $\mathcal{L}(\mathscr{X}(\mathscr{M}, G))$ of all bounded module maps of $\mathscr{X}(\mathscr{M}, G)$ to itself is a von Neumann algebra.

Definition 2.13. The abstract crossed product $\mathscr{A} \mathscr{R}(\mathscr{M}, G, \alpha, \omega)$ of $\mathscr{M}$ by $\alpha$ and $\omega$ is the von Neumann subalgebra of $\mathcal{L}(\mathscr{K}(\mathscr{H}, G))$ generated by the operators $\left\{\pi_{l}(x): x \in \mathcal{K}(\mathscr{H}, G)\right\}$.

In order to show that 2.13 does indeed give an alternate definition of the crossed product we need the following observations. From the comments preceeding 2.13, $\mathscr{X}(\mathscr{M}, G)$ is an Hermitian $\mathscr{M}$-rigged $\mathbb{C}$-module. Thus if, $\pi$ is any representation of $\mathscr{M}$ on a Hilbert space $\mathscr{H}$, Rieffel's construction provides (i) a pre-inner-product structure on the algebraic tensor product on $\mathscr{X}(\mathscr{M}, G) \otimes \mathscr{H}$; (ii) a representation $\rho$ of $\mathcal{L}(\mathscr{X}(\mathscr{M}, G))$ on the completion $\overline{\mathcal{H}}$ of $\mathscr{X}(\mathscr{M}, G) \otimes \mathscr{H}$, determined by $\rho(T)(\tau \otimes \xi)=T \tau \otimes \xi$ for $\tau \in \mathscr{X}(\mathscr{M}, G)$ $T \in \mathcal{L}(\mathscr{X}(\mathscr{M}, G)), \xi \in \mathscr{H}$. We will denote the restriction of $\rho$ to $\mathscr{A} \mathscr{R}(\mathscr{M}, G$, $\alpha, \omega)$ by $\{\bar{\pi}, \overline{\mathcal{H}}\}$. Finally, if $\{\pi, \mathscr{H}\}$ is a faithful normal representation of $\mathscr{M}$ on $\mathscr{H}$, we may consider the p.c.s. $\{\pi(\mathscr{M}), G, \pi \alpha, \pi \circ \omega\}$ acting on $L^{2}(G ; \mathscr{H})$, where $(\pi \alpha)_{g}=\pi \circ \alpha_{g} \circ \pi^{-1}$. 
Theorem 2.14. Let $\{\pi, \mathscr{H}\}$ be a faithful normal representation of $\mathscr{M}$ on $\mathcal{H}$, and $\{\bar{\pi}, \overline{\mathcal{H}}\}$ be as above. Then

i) $\bar{\pi}$ is a faithful normal representation on $\overline{\mathcal{H}}$,

ii) $\{\bar{\pi}(\mathcal{A} \mathscr{R}(\mathscr{M}, G, \alpha, \omega)), \overline{\mathcal{H}}\}$ is unitarily equivalent with $\{\mathscr{R}(\pi(\mathscr{M}), G$, $\left.\pi \alpha, \pi \circ \omega), L^{2}(G, \mathcal{H})\right\}$.

Proof. i) For $\tau \in \mathscr{X}(\mathscr{M}, G), \xi \in \mathscr{H}$, we have $\|\tau \otimes \xi\|^{2}=\left\|\pi\left(\langle\tau, \tau\rangle_{\mathscr{M}}^{1 / 2}\right) \xi\right\|^{2}$, so for $T \in \mathcal{L}(\mathscr{K}(\mathscr{M}, G))$ we have

$$
\|\rho(T) \tau \otimes \xi\|^{2}=\left\|\pi\left(\langle T \tau, T \tau\rangle_{\mathscr{M}}^{1 / 2}\right) \xi\right\|^{2}
$$

Thus $\rho$ (and hence $\bar{\pi}$ ) is faithful whenever $\pi$ is. The normality of $\rho$ (and so of $\bar{\pi})$ follows directly from the identification of the predual of $\mathcal{L}(\mathscr{X}(\mathscr{M}, G))$ given in [17].

ii) To see the unitary equivalence we will need a preparatory result.

Lemma 2.15. $\quad(\mathcal{K}(\mathscr{M}, G)) \otimes \mathscr{H}$ is dense in $\mathscr{X}(\mathscr{H}, G) \otimes \mathscr{H}$.

Proof. From the construction of the inner product on $\mathscr{X}(\mathscr{M}, G)$ given in [17], we see that for $\tau, \tau^{\prime} \in \mathscr{X}(\mathscr{M}, G),\left\langle\tau, \tau^{\prime}\right\rangle_{\mathscr{M}}$ is in the $\sigma$-weak closure of $\left\{\left\langle\iota(x), \tau^{\prime}\right\rangle_{\mathscr{M}}: x \in \mathcal{K}(\mathscr{M}, G)\right\}$. Thus if $\sum_{j=1}^{n} \tau_{j} \otimes \xi_{j} \in \mathscr{X}(\mathscr{M} ; G) \otimes \mathscr{H}$ is orthogonal to $\iota(\mathcal{K}(\mathscr{H} ; G) \otimes \mathscr{H}$ we have, for each $k=1, \cdots, n$

$$
0=\left(\iota(x) \otimes \xi_{k}, \sum_{j=1}^{n} \pi_{j} \otimes \xi_{j}\right)=\sum_{j=1}^{n}\left(\pi\left(\left\langle\iota(x), \tau_{j}\right\rangle \mathscr{M}_{M}\right) \xi_{k}, \xi_{j}\right)
$$

for all $x \in \mathcal{K}(\mathscr{M}, G)$. But then by the above remark, we see that for each $k, \sum_{j=1}^{n}\left(\pi\left(\left\langle\tau_{k}, \tau_{j}\right\rangle \mathscr{M}_{\mathcal{M}}\right) \xi_{k}, \xi_{j}\right)=0$. Summing over all $k$, we finally have

$$
0=\sum_{j, k=1}^{n}\left(\pi\left(\left\langle\tau_{k}, \tau_{j}\right\rangle_{\mathscr{M}}\right) \xi_{k}, \xi_{j}\right)=\left\|\sum_{j=1}^{n} \tau_{j} \otimes \xi_{j}\right\|^{2},
$$

and the assertion is established.

Q.E.D.

Proof of 2.14 (ii). We define a map $U: \iota(\mathcal{K}(\mathscr{M}, G)) \otimes \mathscr{H} \rightarrow L^{2}(G, \mathscr{H})$ by $U(\iota(x) \otimes \xi)=\pi\left(x(g) \omega\left(g^{-1}, g\right)\right) \xi$. We have

$$
\begin{aligned}
& (U(\iota(x) \otimes \xi), U(\iota(y) \otimes \eta)) \\
= & \int_{G}\left(\pi\left(y(g)^{*} x(g)\right) \xi, \eta\right) d g \\
= & \left(\pi\left(\langle\iota(x), \iota(y)\rangle_{\mathcal{M}}\right) \xi, \eta\right) \\
= & (\iota(e) \otimes \xi, \iota(y) \otimes \eta)
\end{aligned}
$$


so that $U$ is an isometry. By 2.14 , and the fact that $U$ clearly has dense range in $L^{2}(G, \mathscr{H})$, we see there is a unitary mapping, also denoted $U$, of $\overline{\mathcal{H}}$ onto $L^{2}(G, \mathscr{H})$ extending the map defined above. Also, for $x, y \in \mathcal{K}(\mathscr{M}, G), \xi \in \mathcal{H}$, we have

$$
\begin{aligned}
& U\left(\bar{\pi}\left(\pi_{l}(x)\right)(\iota(y) \otimes \xi)\right)(g)=U(\iota(x y) \otimes \xi)(g) \\
= & \pi\left(\int_{G} \alpha_{g}^{-1}\left(\omega\left(g h, h^{-1}\right)\right) \omega\left(g^{-1}, g\right) \alpha_{h}(x(g h)) y\left(h^{-1}\right) d h\right) \xi
\end{aligned}
$$

and (denoting $\pi \alpha$ by $\beta, \pi \circ \omega$ by $\nu$ )

$$
\begin{aligned}
& \left(\left(\int_{G} \lambda^{\nu}(h) I^{\beta}(\pi(x(h))) d h\right) U(\iota(y) \otimes \xi)\right)(g) \\
= & \int_{G} \nu\left(g^{-1}, h\right)\left(I^{\beta}(\pi(x(h))) U(\iota(y) \otimes \xi)\right)\left(h^{-1} g\right) d h \\
= & \int_{G} \nu\left(g^{-1}, h\right) \beta_{g^{-1}}(\pi(x(h))) \pi\left(y\left(h^{-1} g\right) \omega\left(g^{-1} h, h^{-1} g\right)\right) \xi d h \\
= & \int_{G} \nu\left(g^{-1}, g h\right) \nu\left(h, h^{-1}\right) \pi\left[\alpha_{h}(x(g h)) y\left(h^{-1}\right)\right] \xi d h \\
= & \pi\left(\int _ { G } \alpha _ { g } ^ { - 1 } \left(\omega\left(g h, h^{-1}\right) \omega\left(g^{-1}, g\right) \alpha_{h}\left(x(g h) y\left(h^{-1}\right) d h\right) \xi .\right.\right.
\end{aligned}
$$

Thus

$$
U \bar{\pi}\left(\pi_{l}(x)\right) U^{*}=\int_{G} \lambda^{\nu}(h) I^{\beta}(\pi(x(h))) d h .
$$

Since such integrals generate $\mathscr{R}(\pi(\mathscr{M}), G, \pi \alpha, \pi \circ \omega)$ the proof is complete.

Q.E.D.

Remark. In case $\omega$ is continuous we may consider instead of $\mathcal{K}(\mathscr{M}, G)$, the space $\mathcal{C}_{C}(\mathscr{M}, G)$ of $\sigma$-strong continuous compactly supported functions from $G$ to $\mathscr{M}$ with the same product, involution and inner product. If we topologize $\mathcal{C}_{c}(\mathscr{M}, G)$ by saying $x_{\mu} \rightarrow x$ if for all $\psi \in \mathscr{M}_{*}^{+}$and all $y \in \mathcal{C}_{c}(\mathscr{M}, G)$, we have $\psi\left(\left\langle x_{\mu}, y\right\rangle_{\mathscr{M}}\right) \rightarrow \psi\left(\langle x, y\rangle_{\mathscr{M}}\right)$. It is then easy to establish that with respect to this topology $\left\{x y: x, y \in \mathcal{C}_{c}(\mathscr{M}, G)\right\}$ is dense, and that $x \rightarrow x^{\#}$ is preclosed as a map on $\mathcal{C}_{c}(\mathscr{M}, G)$. Thus $\mathcal{C}_{c}(\mathscr{H}, G)$ appears as an exact analogue of a left Hilbert algebra in the module context. The "left ring" of $\mathcal{C}_{c}(\mathscr{M}, G)$ is nothing but the abstract crossed product. It is reasonable to hope that at least a part of the Tomita-Takesaki theory of Hilbert algebras might persist in this situation, and in case $\mathscr{M}$ is abelian this has been worked out, in a slightly different context, in [10]. This author has not investigated 
Tomita-Takesaki theory in this context. Nevertheless one might expect there should be an "unbounded expectation" from $\mathscr{R}(\mathscr{M}, G, \alpha, \omega)$ to $\mathscr{M}$ as a generalization of a weight; the consequences of such an expectation can, to a large extent, be imputed from the results of the next section.

\section{§3 Dual Weights on Crossed Products}

Our object here is to produce, in a canonical way, for each p.c.s. $\{\mathscr{M}, G, \alpha, \omega\}$ and each faithful normal semifinite (f.n.s.f) weight $\phi$ on $\mathscr{M}$, a (f.n.s.f) weight $\tilde{\phi}$ on $\mathscr{R}(\mathscr{M}, G, \alpha, \omega)$ which is dual to $\phi$. The duality is expressed in terms of a formula (Proposition 3.5) giving the value of $\tilde{\phi}$ on certain elements of $\mathscr{R}(\mathscr{M}, G, \alpha, \omega)$ in terms of $\phi$, and the relation of the modular objects of $\tilde{\phi}$ to those of $\phi$ (Proposition 3.7). The treatment we give here differs from that of [7] only in that the cocycle $\omega$ must be introduced, and that we are obliged to consider classes of Borel functions from $G$ to $\mathscr{M}$ rather than continuous functions. The construction is also the obvious adaption of that of Section 2 to the situation where a particular f.n.s.f. weight on $\mathscr{M}$ is given. Because of the close similarity of what follows to the results of [7] and to Section 2 we will limit ourselves to stating definitions and Theorems, omitting proofs.

Throughout this section, $\{\mathscr{M}, G, \alpha, \omega\}$ will be a fixed p.c.s., and $\phi$ a fixed f.n.s.f. weight on $\mathscr{M}$. We will denote by $\left\{\pi_{\phi}, \mathcal{H}_{\phi}\right\}$ the associated representation of $\mathscr{M}$, and $\Lambda_{\phi}: \mathfrak{n}_{\phi}=\left\{x \in \mathscr{M}: \phi\left(x^{*} x\right)<\infty\right\} \rightarrow \mathcal{H}_{\phi}$ the canonical injection. By $\left\{\sigma_{t}^{\phi}, t \in \mathbb{R}\right\}, \Delta_{\phi}, J_{\phi}, S_{\phi}$ respectively, we mean the modular automorphism group, modular operator, unitary involution, and sharp operation, respectively, derived from $\phi$; thus $\pi_{\phi}\left(\sigma_{t}^{\phi}(x)\right)=\Delta_{\phi}^{i t} \pi_{\phi}(x) \Delta_{\phi}^{-i t},(x \in \mathscr{M})$ and $S_{\phi}$ $=J_{\phi} \Delta_{\phi}^{1 / 2}=\Delta_{\phi}^{1 / 2} J_{\phi}$ (see [22]).

We set $\phi_{g}=\phi \circ \alpha_{g}$; the modular objects associated to $\phi_{g}$ will be denoted $\left\{\pi_{g} \mathcal{H}_{g}\right\}, \Lambda_{g}, \mathfrak{n}_{g}, \sigma^{g}, \Delta_{g}, J_{g}, S_{g}$ respectively. We will also need to consider the so-called relative modular objects i.e. $S_{g, h}=J_{g, h}\left(\Delta_{h}^{g}\right)^{1 / 2}, u_{t}^{h, g}=\left(D \phi_{h}, D \phi_{g}\right)_{t}$; for the definitions and properties of these operators, we refer to [1] and [7]. The symbols $\{\pi, \mathscr{H}\}, A, \mathfrak{n}$ etc., without subscript, will refer to the original weight $\phi$ (the subscript should be $e$ ).

Definition 3.1. $\tilde{\mathscr{M}}$ is the linear space of equivalence classes of norm bounded, compactly supported, Borel functions $x: G \rightarrow \mathscr{M}$ such that

i) $x(g) \in \mathfrak{n}_{g}^{*} \cap \mathfrak{n}, g \in G$, where $\mathfrak{n}=\mathfrak{n}_{e}$.

ii) the functions $g \rightarrow \phi\left(x(g)^{*} x(g)\right)$ and $g \rightarrow \phi_{g}\left(x(g) x(g)^{*}\right)$ are integrable, 
where the equivalence is given by $x \sim y$ if $x(g)=y(g)$ a.e.

Remark. Since $\mathscr{H}_{*}$ is separable, there is a countable family $\left\{\psi_{n}: n \in \mathbb{Z}_{+}\right\}$ $\subseteq \mathscr{M}_{*}^{+}$with $\phi=\sup \psi_{n}$. Thus for any Borel function $g \rightarrow y(g) \in \mathscr{M}^{+}, g \rightarrow$ $\phi(y(g))$ is Borel.

Definition 3.2. Define a product and an involution on $\tilde{\mathscr{M}}$ by

i) $(x y)(g)=\int_{G} \alpha_{g}^{-1}\left(\omega\left(g h, h^{-1}\right)\right) \alpha_{h}(x(g h)) y\left(h^{-1}\right) d h$

ii) $x^{\sharp}(g)=\alpha_{g}^{-1}\left(x\left(g^{-1}\right)^{*}\right) \omega\left(g^{-1}, g\right)^{*} \delta_{G}\left(g^{-1}\right)$.

for $x, y \in \tilde{\mathscr{M}}$. (As in Section 2, we confuse functions with function classes).

If, as in [7], we define a map $\tilde{\Lambda}: \tilde{\mathscr{M}} \rightarrow L^{2}(G ; \mathcal{H})$ by $(\tilde{\Lambda}(x))(g)$ $=\Lambda\left(x(g) \omega\left(g^{-1}, g\right)\right), x \in \tilde{\mathscr{M}}$, and denote by $\tilde{\mathfrak{A}}$ the image of $\tilde{\mathscr{M}}$, then $\tilde{\mathfrak{A}}$ inherits a product and involution from $\tilde{\mathscr{M}}$ via $\tilde{\Lambda}$, and an inner product as a subspace of $L^{2}(G ; \mathscr{H})$. It should be noted that, with the notations of Section $2, \tilde{\mathscr{M}}$ is a subspace of $\mathcal{K}(\mathscr{M}, G)$, and the inner product on $\widetilde{\mathfrak{A}}$ satisfies

$$
(\tilde{\Lambda}(x), \tilde{\Lambda}(y))=\phi\left(\langle x, y\rangle_{\mathscr{M}}\right), \quad x, y \in \tilde{\mathscr{M}}
$$

The following result is, in effect, an amalgamation of Propositions 3.2, 3.3 and Theorem 3.4 of [7].

Theorem 3.3. Let $\tilde{\mathfrak{A}}$ have the structure as defined above. Then

i) $\widetilde{\mathfrak{A}}$ is a left Hilbert algebra, and is a dense subspace of $L^{2}(G, \mathcal{H})$,

ii) the left ring $\mathcal{R}_{l}(\widetilde{\mathfrak{U}})$ of $\widetilde{\mathfrak{U}}$ coincides with the von Neumann algebra $\mathscr{R}(\pi(\mathscr{M}), G, \pi \alpha, \pi \circ \omega)$, where $\pi=\pi_{e}$, on $L^{2}(G, \mathscr{H})$. Further, for $x \in \mathscr{M}$ we have

$$
\pi_{l}(\tilde{\Lambda}(x))=\int_{G} \lambda^{\pi \circ \omega}(g) I^{\pi \omega}(x(g)) d g .
$$

Definition 3.4. The f.n.s.f. weight on $\mathscr{R}(\mathscr{M}, G, \alpha, \omega)$ determined by the isomorphism of this algebra with $\mathcal{R}_{l}(\widetilde{\mathfrak{A}})$ will be denoted $\tilde{\phi}$ and referred to as the dual weight of $\phi$.

For each norm bounded, Borel, compactly supported map $x: G \rightarrow \mathscr{M}$, let $\tilde{x} \in \mathscr{R}(\mathscr{M}, G, \alpha, \omega)$ denote the operator $\int_{G} \lambda^{\omega}(g) I^{\alpha}(x(g)) d g$. Noting that for such maps $x$ and $y$, the product $x y: G \rightarrow \mathscr{M}$ is in fact continuous (cf. [7], Proposition 3.2), the evaluation $(x y)(e)$ is well defined. Part of the duality between $\phi$ and $\tilde{\phi}$ is then expressed in terms of

Proposition 3.5. Let $x, y: G \rightarrow \mathscr{M}$ be Borel, norm-bounded and of compact 
support, and set $\tilde{x}=\int_{G} \lambda^{\omega}(g) I^{\omega}(x(g)) d g$, and define $\tilde{y}$ in the analogous manner. Then we have

$$
\begin{aligned}
\tilde{\phi}\left(\tilde{y}^{*} \tilde{x}\right) & =\phi\left(\langle x, y\rangle_{\mathscr{H}}\right)=\phi\left(\left(y^{\sharp} x\right)(e)\right) \\
& =\int_{G} \phi\left(y(h)^{*} x(h)\right) d h .
\end{aligned}
$$

Proof. Trivial from the definition of $\tilde{\phi}$ and Theorem 3.3.

Definition 3.6. A pair $(\phi, \tilde{\phi})$ of f.n.s.f. weights will be said to satisfy the Plancherel property, if the conclusions of Proposition 3.5 for every pair of Borel, norm-bounded, compactly supported maps $x, y: G \rightarrow \mathscr{M}$ i.e.

$$
\tilde{\phi}\left(\tilde{y}^{*} \tilde{x}\right)=\int_{G} \phi\left(y(g)^{*} x(g)\right) d g
$$

We shall see, in [21], that the existence of a pair of weights satisfying the Plancherel property is characteristic of crossed products (among all "extensions" of $\mathscr{M})$.

We now turn to the problem of identifying the modular objects associated to $\tilde{\phi}$, and consequently the commutant of $\mathscr{R}(\pi(\mathscr{M}), G, \pi \alpha, \pi \circ \omega)$. For the first result, we need some additional terminology

i) for $x \in \mathfrak{n}_{g}^{*} \cap \mathfrak{n}_{h}$, set $S_{g, h}\left(\Lambda_{h}(x)\right)=\Lambda_{g}\left(x^{*}\right)$,

ii) for $x \in \mathfrak{n}_{g}$, set $U_{h, g}\left(\Lambda_{g}(x)\right)=\Lambda_{h}\left(\alpha_{h^{-1}}(x)\right)$,

iii) for $g \in G$, let $W(g)$ be the canonical cone-preserving unitary on $\mathscr{H}=\mathscr{H}_{e}$ which implements $\alpha_{g}$ in the representation $\pi=\pi_{e}$ (i.e. $W(g) \pi(x) W(g)^{*}$ $\left.=\pi\left(\alpha_{g}(x)\right) x \in \mathscr{M}\right)$. (See [9] for the definition, and [7] for additional properties.) Note that $S_{g, h}$ above is a core of the relative modular object $S_{g, h}$ referred to previously.

Lemma 3.6. Let $\tilde{S}, \tilde{\Delta}, \widetilde{J}$ be the sharp operation, modular operator and unitary involution associated to $\tilde{\phi}$. Then, for $\xi \in \tilde{\mathfrak{A}}$ we have

i) $(\tilde{S \xi})(g)=\delta_{g}\left(g^{-1}\right) U_{e, g} S_{g, e} \pi\left(\omega\left(g, g^{-1}\right)^{*}\right) \xi\left(g^{-1}\right)$,

ii) $(\tilde{\Delta} \xi)(g)=\delta_{G}(g) \Delta_{e}^{g} \xi(g)$,

iii) $(\widetilde{J} \xi)(g)=\delta_{G}(g)^{-1 / 2} J W\left(g^{-1}\right) \pi\left(\omega\left(g, g^{-1}\right)^{*}\right) \xi\left(g^{-1}\right)$ $=\delta_{G}(g)^{-1 / 2} \pi\left(\omega\left(g^{-1}, g\right)\right) J W\left(g^{-1}\right) \xi\left(g^{-1}\right)$.

Proof. See [7] (or compute directly).

Lemma 3.6 has two immediate consequences; the computation of the action of the modular automorphism group $\left\{\tilde{\sigma}_{t}: t \in \boldsymbol{R}\right\}$ of $\tilde{\phi}$, and the 
computation of the generators of the commutant of the crossed product. For convenience we define an action $\beta$ of $G$ on $\pi(\mathscr{M})$, and a 2-cocycle $\nu$ by

$$
\beta_{g}=\pi \circ \alpha_{g} \circ \pi^{-1}, \quad \nu(g, h)=\pi(\omega(g, h)) .
$$

Theorem 3.7. The action of $\left\{\tilde{\sigma}_{t}: t \in \mathbb{R}\right\}$ on the generators of the crossed product $\mathscr{R}(\pi(\mathscr{M}), G, \beta, \nu)$ is given by

$$
\begin{aligned}
\tilde{\sigma}_{t}\left(I^{\beta}(x)\right) & =I^{\beta}\left(\sigma_{t}(x)\right), \\
\tilde{\sigma}_{t}\left(\lambda^{\nu}(g)\right) & =\delta_{G}(g)^{i t} I^{\beta}\left(u_{t}^{e, g^{-1}}\right) \lambda^{\nu}(g) \\
& =\delta_{G}(g)^{i t} \lambda^{\nu}(g) I^{\beta}\left(u_{t}^{g, e}\right) .
\end{aligned}
$$

Theorem 3.8. The commutant of $\mathscr{R}(\pi(\mathscr{M}), G, \beta, \nu)$ on $L^{2}(G, \mathcal{H})$ is generated by the operators

$$
\begin{aligned}
& \left(\pi^{\prime}(a) \xi\right)(g)=a \xi(g), \quad a \in \pi(\mathscr{M})^{\prime}, \\
& \left(\tilde{\rho}^{\omega}(h) \xi\right)(g)=\delta_{G}(h)^{1 / 2} \pi\left(\omega\left(h, h^{-1} g^{-1}\right)^{*}\right) W(h) \xi(g h) .
\end{aligned}
$$

For the proofs of these theorems, we refer to [7]; the computation using Lemma 3.6 is also rather easy.

The computation of the commutant of $\mathscr{R}(\pi(\mathscr{M}), G, \alpha, \omega)$ above allows us to exhibit the crossed product as the fixed point subalgebra of $\mathscr{M} \otimes \mathcal{L}\left(L^{2}(G)\right)$ under an appropriate action of $G$. In general, this action is not a tensor product of actions of $G$.

Let $\{\mathscr{M}, G, \alpha, \omega\}$ be a p.c.s., with $\mathscr{M}$ acting on $\mathscr{H}$. Define, for $h \in G$, $\xi \in L^{2}(G ; \mathscr{H})$,

$$
\left(\rho^{\omega}(h) \xi\right)(g)=\delta_{G}(h)^{1 / 2} \alpha_{h^{-1}}\left(\omega\left(h, h^{-1} g^{-1}\right)^{*}\right) \xi(g h) .
$$

We note that when $\omega$ takes scalar values, $\rho^{\omega}$ is the identity tensored with the right regular projective $\bar{\omega}$-representation.

Theorem 3.9. The crossed product $\mathscr{R}(\mathscr{M}, G, \alpha, \omega)$ on $L^{2}(G ; \mathcal{H})$ coincides with the fixed point subalgebra of $\mathscr{M} \otimes \mathcal{L}\left(L^{2}(G)\right)$ under the action of $g \in G \rightarrow$ $\alpha_{g} \otimes 1 \rho \operatorname{Ad} \rho^{\omega}(g)$ of $G$.

Proof. We may assume that $\mathscr{M}$ acts on the Hilbert space derived from some f.n.s.f. weight on $\mathcal{M}$, and that $\alpha_{h}$ is implemented by $W(h)$, as above, on this space. Then $\left(\alpha_{g} \otimes 1\right) \circ A d \rho^{\omega}(g)$ is implemented by $(W(g) \otimes 1) \rho^{\omega}(g)$ $=\tilde{\rho}^{\omega}(g)$ (notation as in 3.8). The indicated fixed point subalgebra is thus: 


$$
\begin{aligned}
& \left(\mathscr{M} \otimes \mathcal{L}\left(L^{2}(G)\right) \cap\left\{\tilde{\rho}^{\omega}(g): g \in G\right\}^{\prime}\right. \\
= & {\left[\left(\mathscr{M}^{\prime} \otimes 1\right) \vee\left\{\tilde{\rho}^{\omega}(g): g \in G\right\}^{\prime \prime}\right]^{\prime} } \\
= & {\left[\mathscr{R}(\mathscr{M}, G, \alpha, \omega)^{\prime}\right]^{\prime}=\mathscr{R}(\mathscr{M}, G, \alpha, \omega) . }
\end{aligned}
$$

Since $\tilde{\rho}^{\omega}(g) \tilde{\rho}^{\omega}(h)=\left(\omega(g, h)^{*} \otimes 1\right) \rho^{\omega}(g h)$ (an easy computation) Ad $\tilde{\rho}^{\omega}(g) \circ \operatorname{Ad} \tilde{\rho}^{\omega}(h)$ $=\operatorname{Ad} \tilde{\rho}^{\omega}(g h)$ on $\mathscr{M} \otimes \mathcal{L}\left(L^{2}(G)\right)$, and thus we do have a representation of $G$ in Aut $\left(\mathscr{H} \otimes \mathcal{L}\left(L^{2}(G)\right)\right.$.

Q.E.D.

From the remarks preceding Theorem 3.9 we have

Corollary 3.10. Suppose $\omega$ takes scalar values. Then the crossed product $\mathscr{R}(\mathscr{M}, G, \alpha, \omega)$ coincides with the fixed point subalgebra of $\mathscr{M} \otimes \mathcal{L}\left(L^{2}(G)\right)$ under the action $g \in G \rightarrow \alpha_{g} \otimes \operatorname{Ad} \rho^{\omega}(g)$, where, for $g \in G, \xi \in L^{2}(G),\left(\rho^{\omega}(g) \xi\right)(h)$ $=\overline{\omega\left(h, h^{-1} g^{-1}\right)} \xi(h g) \delta_{G}(h)^{1 / 2}$.

\section{\$4. Automorphisms of Crossed Products}

Throughout $\{\mathscr{M}, G, \alpha, \omega\}$ will denote a fixed p.c.s, and $\mathscr{I}=\mathscr{R}(\mathscr{M}, G, \alpha, \omega)$. Suppose $\beta_{0} \in \operatorname{Aut}(\mathscr{M})$; we shall be concerned with the problem of determining when there exists $\beta \in \operatorname{Aut}(\mathcal{R})$ with $\beta\left(I^{\alpha}(x)\right)=I^{\alpha}\left(\beta_{0}(x)\right)$. Such an automorphism $\beta$ will be termed a lifting or extension of $\beta_{0}$ to $\Re$. It is clear that all inner automorphisms on $\mathscr{M}$ admit extensions to $\mathscr{N}$; by Theorem 3.7 all modular automorphism groups admit liftings which are themselves modular automorphism groups; clearly the automorphisms $\alpha_{g}(g \in G)$ admit liftings Ad $\lambda^{\omega}(g)$, although it is not necessarily true that these liftings form a group. It will be convenient to distinguish the following special kind of liftings.

Definition 4.1. Let $\beta_{0} \in$ Aut (M). A strong lifting $\beta$ of $\beta_{0}$ is an extension of $\beta_{0}$ to $\mathcal{I}$ satisfying the additional property that $\beta\left(\lambda^{\omega}(g)\right) \in I^{\alpha}(\mathscr{M}) \lambda^{\omega}(g)$ for all $g \in G$.

Our first result concerns strong liftings of the identity automorphism of $\mathscr{M}$, and characterizes them in terms of $Z_{a}^{1}(G, \mathcal{U}(\mathscr{Z}(\mathscr{M})))$. The analogue of this result for semi-direct products of groups is well known, [14]; see also [24].

Theorem 4.2. i) For each $\rho \in Z_{\alpha}^{1}(G, \mathcal{U}(\mathcal{Z}(\mathscr{M})))$ there is a strong lifting $\tau_{\mathrm{p}}$ of the identity automorphism of $\mathscr{M}$, satisfying $\tau_{\rho}\left(\lambda^{\omega}(g)\right)=I^{\omega}(\rho(g)) \lambda^{\omega}(g)$ for all $g \in G$. The map $\rho \rightarrow \tau_{\rho}$ is a representation of $Z_{\alpha}^{1}(G, \mathcal{U}(\mathcal{Z}(\mathscr{M})))$ in $\operatorname{Aut}(\mathcal{I})$.

ii) Every strong liftiug of the identity is of the form $\tau_{\rho}$ for some 
$\rho \in Z_{a}^{1}(G, \mathcal{U}(\mathcal{Z}(\mathscr{M})))$.

iii) $\rho_{1}, \rho_{2} \in Z_{\alpha}^{1}(G, \mathcal{U}(\mathcal{Z}(\mathscr{M})))$ are cohomologous if and only if $\tau_{\rho_{1}}=\operatorname{Ad} I^{\alpha}(u) \circ \tau_{\rho_{2}}$ for some $u \in \mathcal{U}(\mathscr{M})$.

Proof. i) We may assume $\mathscr{M}$ acts on $\mathcal{H}$ and $\mathscr{N}$ acts on $L^{2}(G ; \mathscr{H})$. For each $\rho \in Z_{a}^{1}(G, \mathcal{U}(\mathscr{Z}(\mathscr{M})))$, define a unitary $T_{\rho}$ on $L^{2}(G ; \mathscr{H})$ by $\left(T_{\rho} \xi\right)(g)$ $=\rho\left(g^{-1}\right)^{*} \xi(g), \xi \in L^{2}(G ; \mathcal{H})$. Clearly $T_{\rho_{1}} T_{\rho_{2}}=T_{\rho_{1} \rho_{2}}$ for $\rho_{1}, \rho_{2} \in Z_{\alpha}^{1}(G, \mathcal{U}(\mathcal{Z}(\mathscr{M})))$, and we may compute

$$
\begin{aligned}
\left(T_{\rho} I^{\alpha}(x) T_{\rho}^{*} \xi\right)(g) & =\rho\left(g^{-1}\right)^{*} \alpha_{g}^{-1}(x) \rho\left(g^{-1}\right) \xi(g) \\
& =\left(I^{\alpha}(x) \xi\right)(g),
\end{aligned}
$$

and

$$
\begin{aligned}
\left(T_{\rho} \lambda^{\omega}(h) T_{\rho}^{*} \xi\right)(g) & =\rho\left(g^{-1}\right)^{*} \omega\left(g^{-1}, h\right) \rho\left(g^{-1} h\right) \xi\left(h^{-1} g\right) \\
& =\alpha_{g}^{-1}(\rho(h)) \omega\left(g^{-1}, h\right) \xi\left(h^{-1} g\right) \\
& =\left(I^{\infty}(\rho(h)) \lambda^{\omega}(h) \xi\right)(g)
\end{aligned}
$$

where we use the cocycle identity $\alpha_{g}(\rho(h)) \rho(g h)^{*} \rho(g)=1$.

ii) If $\beta$ is a strong lifting of the identity automorphism, define $\rho(g)$ by $\beta\left(\lambda^{\omega}(g)\right)=I^{\alpha}(\rho(g)) \lambda^{\omega}(g), g \in G$. Since $I^{\alpha}\left(\alpha_{g}(x)\right)=\beta\left(\lambda^{\omega}(g) I^{\alpha}(x) \lambda^{\omega}(g)^{*}\right)$ $=\rho(g) \lambda^{\omega}(g) I^{\omega}(x) \lambda^{\omega}(g)^{*} \rho(g)^{*}=\rho(g) I^{\alpha}\left(\alpha_{g}(x)\right) \rho(g)^{*}$, for all $x \in \mathscr{M}$ and $g \in G$, we see $\rho(g) \in \mathcal{U}(\mathscr{L}(\mathscr{M}))$.

Clearly, $\rho(e)=1$, and we have;

$$
\begin{aligned}
& I^{\omega}\left(\rho(g) \alpha_{g}(\rho(h)) \omega(g, h)\right) \lambda^{\omega}(g h) \\
= & I^{\omega}\left(\rho(g) \alpha_{g}(\rho(h))\right) \lambda^{\omega}(g) \lambda^{\omega}(h) \\
= & I^{\omega}(\rho(g)) \lambda^{\omega}(g) I^{\omega}(\rho(h)) \lambda^{\omega}(h) \\
= & \beta\left(\lambda^{\omega}(g) \lambda^{\omega}(h)\right) \\
= & I^{\infty}(\rho(g h) \omega(g, h)) \lambda^{\omega}(g h),
\end{aligned}
$$

so that $\rho(g) \alpha_{g}(\rho(h))=\rho(g h)$. Since $g \rightarrow \rho(g)$ is clearly Borel, $\rho \in Z_{\alpha}^{1}(G$, $\mathcal{U}(\mathscr{Z}(\mathscr{M})))$ and $\beta=\tau_{\rho}$.

iii) If $\tau_{\rho_{1}}=\operatorname{Ad} I^{\alpha}(u) \circ \tau_{\rho_{2}}$ for $u \in \mathcal{U}(\mathscr{M})$, then $\tau_{\rho_{1}}\left(I^{\alpha}(x)\right)=I^{\alpha}(x)=\tau_{\rho_{2}}\left(I^{\alpha}(x)\right)$ forces $u \in \mathcal{U}(\mathscr{Z}(\mathscr{M}))$. But then we have $I^{\omega}\left(\rho_{1}(g)\right)=\tau_{\rho_{1}}\left(\lambda^{\omega}(g)\right) \lambda^{\omega}(g)^{*}$ $=I^{\alpha}(u) I^{\alpha}\left(\rho_{2}(g)\right) \lambda^{\omega}(g) I^{\infty}(u) * \lambda^{\omega}(g)^{*}=I^{\alpha}\left(u \rho_{2}(g) \alpha_{g}(u)^{*}\right.$, and thus $\rho_{2}(g)=(\partial u)(g) \rho_{1}(g)$ for all $g \in G$.

Conversely if $\rho_{2}(g)=(\partial u)(g) \rho_{1}(g)$, for all $g \in G$, then a trivial computation shows $\tau_{\rho_{1}}=\operatorname{Ad} I^{\infty}(u) \circ \tau_{\rho_{2}}$.

Q.E.D.

Remark. i) The theorem identifies $H_{\alpha}^{1}(G, \mathcal{U}(\mathscr{Z}(\mathscr{M})))$ as the classes 
of strong extensions of the identity with respect to the canonical action of $\mathcal{U}(\mathcal{Z}(\mathscr{M}))$ on $\operatorname{Aut}(\mathscr{N})$.

ii) If $G$ is abelian, there is a canonical injection of the dual $\hat{G}$ of $G$ in $Z_{\omega}^{1}(G, \mathcal{U}(\mathscr{Z}(\mathscr{M})))$. The restriction of the representation $\tau$ to $\hat{G}$ is nothing but the dual action of $G$ defined in [23] (for the case $\omega=\imath$ ).

We now turn to the problem of finding the strong liftings of an arbitrary $\beta_{0} \in \operatorname{Aut}(\mathscr{M})$, or if indeed there are any. We use the standard notation for group commutators $[\alpha, \beta]=\alpha \beta \alpha^{-1} \beta^{-1}$.

Theorem 4.3. An automorphism $\beta_{0}$ of $\mathscr{M}$ admits a strong lifting to $\mathscr{N}$ if and only if there is a Borel map $g \rightarrow u_{g} \in \mathcal{U}(\mathscr{M})$ such that

i) $\left[\beta_{0}, \alpha_{g}\right]=\operatorname{Ad} u_{g}, \quad g \in G$,

ii) $\beta_{0}(\omega(g, h))=u_{g} \alpha_{g}\left(u_{h}\right) u_{g h}^{*} \omega(g, h)=(\partial u)(g, h) \omega(g, h) ; g, h \in G$.

If the conditions are satisfied, then there is a strong lifting $\beta$ of $\beta_{0}$ with $\beta\left(\lambda^{\omega}(g)\right)=I^{\infty}\left(u_{g}\right) \lambda^{\omega}(g)$.

Proof. i) Suppose $\beta_{0}$ admits a strong lifting $\beta$, and we define $u_{g}$ $\in \mathcal{U}(\mathcal{M})$ by $\beta\left(\lambda^{\omega}(g)\right)=I^{\omega}\left(u_{g}\right) \lambda^{\omega}(g)$. Then we may compute

$$
\beta\left(\lambda^{\omega}(g) I^{\alpha}(x) \lambda^{\omega}(g)^{*}\right)=I^{\omega}\left(u_{g} \alpha_{g} \circ \beta_{0}(x) u_{g}^{*}\right),
$$

and

$$
\beta\left(I^{\alpha}\left(\alpha_{g}(x)\right)\right)=I^{\infty}\left(\beta_{0} \circ \alpha_{g}(x)\right) \quad \text { for } x \in \mathscr{M}, g \in G .
$$

Thus $\left[\beta_{0}, \alpha_{g}\right]=\operatorname{Ad} u_{g} . \quad$ Furthermore

$$
\begin{aligned}
\beta\left(\lambda^{\omega}(g) \lambda^{\omega}(h)\right) & =\beta\left(I^{\omega}(\omega(g, h)) \lambda^{\omega}(g h)\right) \\
& =I^{\omega}\left(\beta_{0}(\omega(g, h)) u_{g h}\right) \lambda^{\omega}(g h) .
\end{aligned}
$$

On the other hand we have

$$
\begin{aligned}
\beta\left(\lambda^{\omega}(g) \lambda^{\omega}(h)\right) & =I^{\alpha}\left(u_{g}\right) \lambda^{\omega}(g) I^{\omega}\left(u_{h}\right) \lambda^{\omega}(h) \\
& =I^{\omega}\left(u_{g} \alpha_{g}\left(u_{h}\right) \omega(g, h)\right) \lambda^{\omega}(g h) .
\end{aligned}
$$

Thus the second condition is also satisfied, and the conditions are necessary for the existence of a strong lifting.

ii) Suppose $\beta_{0} \in \operatorname{Aut}(\mathscr{M})$ satisfies (i) and (ii) for some family $\left\{u_{g}, g \in G\right\}$ $\subseteq \mathcal{U}(\mathscr{M})$. We suppose $\mathscr{M}$ acts on the Hilbert space $\mathscr{H}$, and that $\beta_{0}$ is implemented on $\mathscr{H}$ by some unitary $b_{0}$. Regarding $\mathscr{I}$ as acting on $L^{2}(G ; \mathscr{H})$, we may define a unitary $b$ by $(b \xi)(g)=u_{g}^{*}-1 b_{0} \xi(g), \xi \in L^{2}(G ; \mathcal{H})$, and compute: 


$$
\begin{aligned}
\left(b I^{\alpha}(x) b^{* \xi} \xi\right)(g) & =u_{g}^{*-1} b_{0} \alpha_{g}^{-1}(x) b_{0}^{*} u_{g^{-1}} \xi(g) \\
& =\operatorname{Ad} u_{g}^{*-1 \circ} \beta_{0} \circ \alpha_{g}^{-1}(x) \xi(g) \\
& =\alpha_{g}^{-1} \circ \beta_{0}(x) \xi(g), \quad(\text { by (i) }), \\
& =\left(I^{\alpha}\left(\beta_{0}(x)\right) \xi\right)(g),
\end{aligned}
$$

and

$$
\begin{aligned}
\left(b \lambda^{\omega}(h) b^{*} \xi\right)(g) & =u_{g}^{*-1} b_{0} \omega\left(g^{-1}, h\right) b_{0}^{*} u_{g^{-1}} \xi\left(h^{-1} g\right) \\
& =\alpha_{g}^{-1}\left(u_{h}\right) \omega\left(g^{-1}, h\right) \xi\left(h^{-1} g\right) \quad \text { (by (ii)) } \\
& =\left(I^{\alpha}\left(u_{h}\right) \lambda^{\omega}(h) \xi\right)(g) .
\end{aligned}
$$

The proof is complete.

Q.E.D.

Remarks. i) The Theorem above guarantees the existence of liftings of modular automorphisms on $\mathscr{M}$ to $\mathscr{\eta}$; the conditions of the theorem are precisely the properties of $g \rightarrow \delta_{G}(g)^{i t} u_{t}^{e, g^{-1}}$ which result from the properties of the Radon derivative $u_{t}^{e, g^{-1}}$ in [7]. (Here notations are as in Section 3, and we have taken $\beta_{0}=\sigma_{t}^{\phi}$ for some f.n.s.f. weight $\phi$ on $\mathscr{M}$. Note $\sigma_{t}^{\phi}(\omega(g, h))$ $=\omega(g, h)$ for all $g, h \in G)$.

ii) The problem of determing in the general case when a strong lifting is inner on $\Omega$ seems very difficult; the author has no satisfactory results in this direction. The reader should however consult [24].

iii) If $v \in \mathcal{Q}(\mathscr{M}), \operatorname{Ad} I^{\infty}(v)$ constitutes a strong lifting of $\operatorname{Ad} v$, with Ad $I^{\omega}(v)\left(\lambda^{\omega}(g)\right)=I^{\alpha}\left(v \alpha_{g}\left(v^{*}\right)\right) \lambda^{\omega}(g)$. Thus the map $g \rightarrow u_{g}$ from 4.3 is a (nonabelian) coboundary. The converse however is false; i.e., if $g \rightarrow u_{g}$ is a coboundary and $\left[\beta_{0}, \alpha_{g}\right]=\operatorname{Ad} u_{g}$ it is not necessarily true that $\beta_{0}$ is even inner.

The final result is concerned with finding necessary conditions for the existence of some lifting of an arbitrary $\beta_{0} \in \operatorname{Aut}(\mathscr{M})$.

Theorem 4.4. Let $\beta_{0} \in \operatorname{Aut}(\mathscr{M})$. If $\beta_{0}$ admits a lifting to $\mathscr{\eta}$, then there is a Borel map $g \in G \rightarrow v_{g} \in \mathcal{U}(\Re)$ such that

i) $I^{\alpha} \circ\left[\beta_{0}, \alpha_{g}\right] \circ I^{\alpha-1}=\operatorname{Ad} v_{g}$ on $I^{\alpha}(\mathscr{M})$, for $g \in G$,

ii) $I^{\alpha}\left(\beta_{0}(\omega(g, h))\right)=v_{g} \lambda^{\omega}(g) v_{h} \lambda^{\omega}(g)^{*} v_{g h}^{*} I^{\alpha}(\omega(g, h))$

$$
=(\partial v)(g, h) I^{\omega}(\omega(g, h)), \quad g, h \in G \text {. }
$$

The proof follows the pattern of the first part of Theorem 4.3; we leave the details to the reader.

Remark. In case $\mathscr{M}$ is abelian, and $G$ is discrete and freely acting, the conditions of Theorem 4.4 may be stated as follows (see [24]); if $\beta_{0}$ admits 
a lifting, then $\left[\beta_{0}, \alpha_{g}\right]$ is in the full group of $G$ for all $g \in G$ and $\left(\beta_{0} \omega\right) \omega^{-1}$ cobounds in $Z_{a}^{2}(G, \mathcal{U}(\mathcal{R}))$.

\section{§5. Removal of Cocycles}

Throughout, $\{\mathscr{M}, G, \alpha, \omega\}$ will denote a fixed p.c.s. We shall be concerned with finding conditions under which we may find another action $\left\{\beta_{g}: g \in G\right\}$ of $G$ on $\mathscr{M}$ such that $\mathscr{R}(\mathscr{M}, G, \alpha, \omega)$ is isomorphic with $\mathscr{R}(\mathscr{M}, G, \beta, \iota)=\mathscr{R}(\mathscr{M}, G, \beta)$. This turns out to be possible whenever $\mathscr{M}$ is a properly infinite factor, or when $G$ is amenable and $\mathscr{M}$ is the $\mathrm{II}_{1}$-hyperfinitefactor. Our most general result covers other situations also.

Theorem 5.1. Let $\mathscr{M}$ and $\mathscr{Q}$ be factors, and $\{\mathscr{M}, G, \alpha, \omega\}$ a p.c.s. Suppose

i) $\mathscr{M} \otimes \mathscr{Q}$ is isomorphic with $\mathscr{M}$,

ii) there is a projective $\omega^{-1}$-representation of $G$ in $\mathscr{Q}$.

Then there is an action $\left\{\beta_{g}: g \in G\right\}$ of $G$ on $\mathscr{M}$ with $\mathscr{R}(\mathscr{M}, G, \alpha, \omega) \otimes \mathscr{Q}$ isomorphic with $\mathscr{R}(\mathcal{M}, G, \beta)$.

Proof. First note that since $\mathscr{M}$ is a factor, $\omega$ has scalar values; thus if $\pi$ is the $\omega^{-1}$ representation of $G$ in $\mathscr{L}, \rho_{g}=\operatorname{Ad} \pi(g)$ defines an action of $G$ on $\mathscr{L}$. If $\kappa: \mathscr{M} \rightarrow \mathscr{M} \otimes \mathscr{Q}$ is the isomorphism in (i), we define an action $\beta_{g}$ of $G$ on $\mathscr{M}$ by $\beta_{g}=\kappa^{-1} \circ\left(\alpha_{g} \otimes \rho_{g}\right) \circ \kappa$; by Proposition $2.3, \mathscr{R}(\mathscr{M}, G, \beta)$ is isomorphic with $\mathscr{R}(\mathscr{M} \otimes \mathscr{Q}, G, \alpha \otimes \rho)$.

If we assume that $\mathscr{M}$ acts on $\mathscr{H}$, and $\mathscr{P}$ acts on $\mathcal{K}$, and that the generators of $\mathscr{R}(\mathscr{M} \otimes \mathscr{Q}, G, \alpha \otimes \rho)$ on $L^{2}(G ; \mathscr{H} \otimes \mathcal{K})$ are

$$
\begin{aligned}
& \left(I^{\infty \otimes P}(x \otimes y) \xi\right)(g)=\alpha_{g}^{-1}(x) \otimes \pi\left(g^{-1}\right) y \pi\left(g^{-1}\right)^{*} \xi(g), \quad x \in \mathcal{M}, y \in \mathcal{P}, \\
& (\lambda(h) \xi)(g)=\xi\left(h^{-1} g\right), \quad h \in G .
\end{aligned}
$$

Define a unitary $U$ on $L^{2}(G: \mathscr{H} \otimes \mathcal{K})$ by

$$
(U \xi)(g)=\left(1 \otimes \pi\left(g^{-1}\right)^{*}\right) \xi(g), \quad \xi \in L^{2}(G ; \mathscr{H} \otimes \mathcal{K})
$$

It is easy to see that $U\left(I^{\kappa \otimes P}(x \otimes y)\right) U^{*}=I^{\alpha}(x) \otimes y(x \in \mathcal{M}, y \in \mathcal{P})$, where we have identified $L^{2}(G ; \mathscr{H} \otimes \mathcal{K})$ with $L^{2}(G ; \mathscr{H}) \otimes \mathcal{K}$ in the canonical manner, and $I^{\alpha}$ has its usual meaning. We may also compute

$$
\begin{aligned}
\left(U \lambda(h) U^{*} \xi\right)(g) & =\left(1 \otimes \pi\left(g^{-1}\right)^{*}\right)\left(1 \otimes \pi\left(g^{-1} h\right)\right) \xi\left(h^{-1} g\right) \\
& =\omega\left(g^{-1}, h\right)(1 \otimes \pi(h)) \xi\left(h^{-1} g\right) .
\end{aligned}
$$

Thus, under the identification of the Hilbert spaces as above, $U(\lambda(h)) U^{*}$ 
$=\lambda^{\omega}(h) \otimes \pi(h)$, where $\lambda^{\omega}$ is as usual. The generators of $U(\mathscr{R}(\mathscr{M} \otimes \mathscr{P}, G$, $\alpha \otimes \rho) V) U^{*}$ on $L^{2}(G ; \mathcal{H}) \otimes \mathcal{K}$ are $I^{\omega}(x) \otimes y, x \in \mathscr{M}, \quad y \in \mathscr{Q}$, and $\lambda^{\omega}(h) \otimes \pi(h)$, $h \in G$. Since $\pi(h) \in \mathscr{Q}$ for all $h$, these operators generated precisely $\mathscr{R}(\mathscr{M}$, $G, \alpha, \omega) \otimes \mathscr{Q}$.

Q.E.D.

Corollary 5.2. Let $\{\mathscr{M}, G, \alpha, \omega\}$ be a p.c.s., with $\mathscr{M}$ a property infinite factor. Then there is an action $\left\{\beta_{g}: g \in G\right\}$ of $G$ on $\mathscr{M}$ such that $\mathscr{R}(\mathscr{M}, G, \alpha, \omega)$ is isomorphic with $\mathcal{R}(\mathscr{M}, G, \beta)$.

Proof. Let $F_{\infty}$ denote the $I_{\infty}$-factor. Since $\mathscr{M}$ is properly infinite, $\mathscr{M} \otimes F_{\infty}$ is isomorphic with $\mathscr{M}$, and $G$ automatically has $\omega^{-1}$-representations in $F_{\infty}$; we apply 5.1 with $\mathcal{Q}=F_{\infty}$. Finally as $\mathcal{R}(\mathscr{M}, G, \alpha, \omega)$ is properly infinite whenever $\mathscr{M}$ is, we are done.

Q.E.D.

For the statement of 5.3, $\mathcal{R}$ denotes the hyperfinite $\mathrm{II}_{1}$-factor.

Corollary 5.3. Let $\mathscr{M}$ be a $I I_{1}-$ factor, $G$ a discrete amenable group, and $\{\mathscr{M}, G, \alpha, \omega\}$ a p.c.s. Suppose that $\mathscr{M} \otimes \mathcal{R}$ is isomorphic with $\mathscr{M}$. Then $\mathscr{R}(\mathscr{M}, G, \alpha, \omega) \otimes \mathscr{R}$ is isomorphic with $\mathscr{R}(\mathscr{M}, G, \beta)$ for some action $\left\{\beta_{g}: g \in G\right\}$ of $G$ on $\mathscr{M}$.

Proof. This follows from 5.1 provided we can guarantee that $G$ has projective $\omega^{-1}$ representation in $\mathcal{R}$. But by the work of Connes [5], the left regular $\omega^{-1}$ representation of $G$ is hyperfinite, and thus by direct integral theory, $G$ has $\omega^{-1}$ representation which are either finite dimensional, or generate $\mathcal{R}$. In either case we are done.

Q.E.D.

Remarks. i) The condition $\mathscr{M} \otimes \mathscr{R} \simeq \mathscr{M}$ is equivalent to a number of other properties e.g., non-commutativity of the algebra of central sequences on $\mathscr{M}$, or noncommutativity of Int $\mathscr{M} /$ Int $\mathscr{M}$. (See [4], [11].) Clearly $\mathscr{R}$ itself has this property; however for discrete amenable $G$ the crossed product $\mathcal{R}(\mathscr{N}, G, \alpha, \omega)$ is already known to a hyperfinite $\mathrm{II}_{1}$-algebra (see [5]).

ii) The theorem and its corollaries admit obvious generalization to the case where $\mathscr{M}$ is not a factor, but $\left\{\alpha_{g}: g \in G\right\}$ acts trivially on the centre of $\mathscr{M}$; reduction to the factor case is possible with the usual techniques of direct integral theory. We omit details.

iii) Interest in crossed products with cocycles clearly reduces primarily to the case where $\mathscr{M}$ is abelian, or the group $G$ is non-amenable. We are currently investigating these situations. 


\section{§6. Examples}

We give non trivial examples of crossed products of abelian von Neumann algebras with cocycles; in particular we show the analogy between this kind of crossed product and the usual extension theory of abelian topological groups, and show how various examples of factors not anti-isomorphic with themselves may be constructed. Unfortunately, no proof of the non-anti-isomorphism via cohomological methods exists (to the authors knowledge); Connes' techniques seem essential here. Finally we give some comments on computing second cohomology groups of various actions; as shown in [15], the techniques of dimension shifting and spectral sequences are available in our context, but these are often insufficient for effective computation.

Theorem 6.1. Let $1 \rightarrow A \rightarrow X \rightarrow G \rightarrow 1$ be an exact sequence of loca!ly compact groups, with $A$ abelian, and let $\mathscr{M}(A), \mathscr{M}(X)$ denote the von Neumann algebras generated by the left regular representations of $A$ and $X$. Then for some action $\left\{\alpha_{g}: g \in G\right\}$ of $G$ on $\mathscr{M}(A)$, and some $\omega \in Z_{a}^{2}(G, \mathcal{U}(\mathscr{M}(A)))$ we have $\mathscr{M}(X)$ isomorphic with $\mathscr{R}(\mathscr{H}(A), G, \alpha, \omega)$.

Proof. From [15] there is an action $\left\{\bar{\alpha}_{g}: g \in G\right\}$ of $G$ on $A$ and an $\bar{\omega} \in Z \frac{2}{\bar{\alpha}}(G, A)$ such that, as Borel groups, $X$ is identified with $A \times G$ under the multiplication

$$
(a, g)(b, h)=\left(a \bar{\alpha}_{g}(b) \bar{\omega}(g, h), g h\right) ;(\alpha, g),(b, h) \in A \times G .
$$

$\bar{\alpha}$ and $\bar{\omega}$ are determined by a (Borel) lifting $g \in G \rightarrow x_{g} \in X$ via $\bar{\alpha}_{g}(a)=x_{g} a x_{g}^{-1}$ and $x_{g} x_{h}=\bar{\omega}(g, h) x_{g h}$. Further, if $d_{A}$ and $d_{G}$ are right Haar measures on $A$ and $G$, then $d_{A} \times d_{G}=d_{X}$ is a right Haar measure on $X$ (viewed as $A \times G$ ).

The algebra $\mathscr{M}(X)$, on $L^{2}\left(G, d_{X}\right)$ is generated by the operators

$$
\begin{gathered}
\left(\lambda^{x}(a, e) \xi\right)(b, h)=\xi\left(a^{-1} b, h\right), \quad a \in A, \\
\left(\lambda^{x}(e, g) \xi\right)(b, h)=\delta_{G}(g)^{1 / 2}\left(\bar{\alpha}_{g}^{-1}\left(\bar{\omega}\left(g, g^{-1} h\right)^{-1} b\right), g^{-1} h\right), \quad g \in G .
\end{gathered}
$$

To determine the crossed product, define unitaries $u_{g}$ on $L^{2}\left(A, d_{A}\right)$ by $\left(u_{g} \xi\right)(h)=\xi\left(\bar{\alpha}_{g}^{-1}(h)\right) \delta_{A}(g)^{1 / 2}$, where $\delta_{A}(g)$ is the (constant) Radon derivative $d_{A} \circ \bar{\alpha}_{g}^{-1} / d_{A}$. We denote by $\alpha_{g}$ the automorphism Ad $u_{g}$ of $\mathscr{M}(A)$,-it is clear that $g \rightarrow \alpha_{g}$ gives an action of $G$ on $\mathscr{M}(A)$ and $\alpha_{g}\left(\lambda^{A}(a)\right)=\lambda^{A}\left(\bar{\alpha}_{g}(\alpha)\right)$. Also define $\omega(g, h)=\lambda^{A}(\bar{\omega}(g, h)) \in \mathcal{U}(\mathscr{H}(A))$; it is trivial that $\omega \in Z_{\alpha}^{2}(G, \mathcal{U}(\mathscr{M}(A)))$.

Using Lemma 2.5 we obtain generators on $L^{2}\left(G ; L^{2}(A)\right)=L^{2}(G \times A)$ $=L^{2}(G) \otimes L^{2}(A)$ of the form 


$$
\begin{gathered}
\tilde{I}\left(\lambda^{A}(a)\right)=\lambda^{A}(a) \otimes 1, \quad a \in A, \\
\left(\tilde{\lambda}^{\omega}(g) \xi\right)(h)=\delta_{G}(g)^{1 / 2} \omega\left(g, g^{-1} h\right) u_{g} \xi\left(g^{-1} h\right), \quad g \in G .
\end{gathered}
$$

On $L^{2}(G \times A)$, the expression for $\tilde{\lambda}^{\omega}(g)$ is

$$
\left(\tilde{\lambda}^{\omega}(g) \xi\right)(b, h)=\delta_{G}(g)^{1 / 2} \xi\left(\bar{\alpha}_{g}^{-1}\left(\omega\left(g, g^{-1} h\right)^{-1} b\right), g^{-1} h\right)
$$

Thus $\lambda^{x} /(\alpha, e)$ coincides with $\widetilde{I}\left(\lambda^{\omega}(a)\right)$ and $\lambda^{x}(e, g)$ coincides with $\tilde{\lambda}^{\omega}(g)$. Q.E.D.

Remarks. i) The assertion of the Theorem remains valid when $A$ is non-abelian, provided $X$ is identified with $A \times G$ with multiplication as defined above, for some action $\bar{\alpha}_{g}$ of $G$ on $A$ and some $\bar{\omega}$ with values in the centre of $A$. In general, however, $X$ need not be of this form.

ii) It can happen that $\omega$ is non-trivial (in $H_{\bar{\alpha}}^{2}(G, A)$ ) while $\omega$ is trivial in $H_{\alpha}^{2}(G, \mathcal{U}(\mathscr{M}(A)))$; see the material following Proposition 6.2.

We turn now to the examples of Connes, [3], of factors $Q_{\lambda, p, \gamma}$ not antiisomorphic with themselves; we present them as crossed products of abelian algebras by discrete groups and cocycles.

We shall need the following notations; $\lambda \in(0,1), p \in Z^{+}$and $r \in T=$ $\{z \in \mathbb{C}:|z|=1\}$ are fixed with $r^{p}=1 . \mathbb{Z}_{p}$ denotes the cyclic group with $p$ elements $\{0,1, \cdots, p-1\}$, and $\mathbb{F}_{2}$ the free group on two generators. Let $X_{p}$ be the direct product, and $\chi_{p}$ the weak direct product, of infinitely many copies of $\mathbb{Z}_{p}(p \geq 2)$. Thus $\chi_{p}$ is a subgroup of $X_{p}$. For $p \geq 3$, let $m_{p}$ be the Haar measure on $X_{p}$; for $p=2$ we consider the measure $\mu_{\lambda}$ on $X_{2}$ which is the product of measures $\tau_{\lambda}$ on $\mathbb{Z}_{2}$, where $\tau_{\lambda}(\{0\})=\lambda / 1+\lambda, \tau_{\lambda}(\{1\})=1 / 1+\lambda$. Finally, $m_{\lambda}$ will denote the measure on $\mathbb{Z}$ with $m_{\lambda}(\{n\})=\lambda^{n}$.

We wish to define actions of $\chi_{p}, \chi_{2}, \mathbb{F}_{2}$ and $\mathbb{Z}$ on the space $(Y, \nu)$ $=\left(X_{p} \times X_{2} \times \mathbb{Z}, m_{p} \times \mu_{\lambda} \times m_{\lambda}\right)$. We denote elements of $Y$ by triples $(x, s, n)$.

(i) The action of $\chi_{p}$ is given by $(x, s, n) \rightarrow(x-y, s, n)$ under $y \in \chi_{p}$.

(ii) $X_{2}$ acts on $X_{2}$ via $s \rightarrow s-t\left(s \in X_{2}, t \in X_{2}\right)$; also if we view the index set in the product defining $X_{2}$ as being $\mathbb{F}_{2}$, then elements of $X_{2}$ are sequences $\left\{s(w): w \in \mathbb{F}_{2}\right\}$ of zeros and ones. Thus $\mathbb{F}_{2}$ acts on $X_{2}$ via $\left(w_{1} s\right)\left(w_{2}\right)=s\left(w_{1}^{-1} w_{2}\right)$, $s \in X_{2}, w_{1}, w_{2} \in \mathbb{F}_{2}$. Since this action of $\mathbb{F}_{2}$ carries $\chi_{2}$ to $\chi_{2}$, the actions of $\chi_{2}$ and $\boldsymbol{F}_{2}$ on $X_{2}$ generate an action of the semidirect product $\chi_{2} \times_{s} \mathbb{F}_{2}$ (with $\boldsymbol{F}_{2}$ acting on $\left.\chi_{2}\right)$ on $X_{2}$. For $g \in \chi_{2} \times_{s} \mathbb{F}_{2}$, let $\psi_{g}$ be the Radon derivative $d \mu_{\lambda} \circ g / d \mu_{\lambda}$; since $\psi_{g}(s) \in\left\{\lambda^{n}: n \in \mathbb{Z}\right\}$ for all $s, \phi_{g}=\log _{\lambda} \psi_{g}$ takes integer values. The action of $\chi_{2} \times{ }_{s} F_{2}$ on $Y$ which we are concerned with is 


$$
(x, s, n) \rightarrow\left(x, g s, n-\phi_{g}(s)\right), \quad g \in \chi_{2} \times{ }_{s} F_{2} .
$$

The action of $\chi_{2} \times_{s} F_{2}$ on $X_{2} \times \mathbb{Z}$ involved here, is nothing but the skew product of the action of $\chi_{2} \times_{s}$ defined on $X_{2}$ (see [13]).

It should be noted that the actions of $\chi_{p}$ and of $\chi_{2} \times_{s} F_{2}$ on $Y$ commute, and both preserve the measure $\nu$.

(iii) The action of $\mathbb{Z}$ on $Y$ is generated by the transformation $(x, s, n) \rightarrow$ $(x-1, s, n-1)$, where 1 denotes the sequence $(1,1, \cdots) \in X_{p}$. We denote by $\sigma$ the automorphism of $L^{\infty}\left(X_{p}, m_{p}\right)$ corresponding to the transformation $x \in X_{p} \rightarrow x-1$ (denoted $\sum$ in [3])

It is trivial to see that the actions of $\chi_{p}$ and $\chi_{2} \times_{s} F_{s}$ commute with that of $\mathbb{Z}$, so that we obtain an action of $G=\mathbb{Z} \times \chi_{p} \times\left(\chi_{2} \times{ }_{s} \mathbb{F}_{2}\right)$ on $Y$. The corresponding action of $G$ on $L^{\infty}(Y, \nu)$ will be denoted $\left\{\alpha_{g}: g \in G\right\}$; it is easy to see that this action is free and ergodic.

Finally, let $\rho \in Z^{1}\left(\chi_{p}, \vartheta\left(L^{\infty}\left(X_{p}, m_{p}\right)\right)\right)$ be the one-cocycle constructed in [3]; the action of $\chi_{p}$ here is that induced by translation on $X_{p}$. In what follows, we regard $\mathcal{U}\left(L^{\infty}\left(X_{p}, m_{p}\right)\right)$ as embedded in $\mathcal{U}\left(L^{\infty}(Y, \nu)\right)$ in the canonical manner.

Proposition 6.2. Let $G=\mathbb{Z} \times \chi_{p} \times\left(\chi_{2} \times{ }_{s} F_{2}\right)$ act on $L^{\infty}(Y, \nu)$ as above. Define $\omega: G \times G \rightarrow \mathcal{U}\left(L^{\infty}(Y, \nu)\right)$ by

$$
\begin{aligned}
\omega((m, r, g),(n, s, h)) & =\prod_{j=m+1}^{n+m} \sigma^{j}\left(\rho_{r}^{*}\right) \otimes 1, & & n \geq 1, \\
& =1, & & n=0, \\
& =\prod_{m+n+1}^{m} \sigma^{j}\left(\rho_{r}\right) \otimes 1, & & n \leq-1 .
\end{aligned}
$$

Then $\omega \in \mathbb{Z}^{2}\left(G, \mathcal{U}\left(L^{\infty}(Y, \nu)\right)\right)$, and $\mathcal{R}\left(L^{\infty}(Y, \nu), G, \alpha, \omega\right)$ is isomorphic with Connes' factor $Q_{\lambda, p, \gamma}$.

Proof. The fact that $\omega$ is a two-cocycle is the result of a long, tedious, but essentially trivial computation, which we leave to the reader.

We now examine the crossed product; it acts on the Hilbert space $l^{2}\left(G ; L^{2}(Y, \nu)\right)$, and is generated by the operators, $I^{\infty}(x), x \in L^{\infty}(Y, \nu), \lambda^{\omega}(0, r, e)$, $r \in \chi_{p}, \lambda^{\omega}(0,0, g), g \in \chi_{2} \times{ }_{s} \mathbb{F}_{2}$, and $\lambda^{\omega}(1,0, e)$. It follows from the form of the cocycle $\omega$, and Proposition 2.2 that $\lambda^{\omega}(0,0, g)$ commutes with both $\lambda^{\omega}(0, r, e)$ and $\lambda^{\omega}(1,0, e)$, while $\lambda^{\omega}(1,0, e) \lambda^{\omega}(0, r, e) \lambda^{\omega}(1,0, e)^{*}=I^{\omega}\left(\sigma\left(\rho_{r}\right) \otimes 1\right) \lambda^{\omega}(0, r, e)$ for all $r \in \chi_{p}$.

Writing these operators in the form given by Lemma 2.5 , acting on the 
Hilbert space $L^{2}\left(X_{p}, m_{p}\right) \otimes L^{2}\left(X_{2}, \mu_{\lambda}\right) \otimes l^{2}(Z) \otimes l^{2}(Z) \otimes l^{2}\left(\chi_{p}\right) \otimes l^{2}\left(\chi_{2} \times_{s} F_{2}\right)$, we obtain generators $(*)$,

$$
\begin{array}{ll}
a \otimes b \otimes c \otimes 1 \otimes \lambda(r) \otimes 1, & a \in L^{\infty}\left(X_{p}, m_{p}\right), b \in L^{\infty}\left(X_{2}, \mu_{\lambda}\right), \\
c \in l^{\infty}(\mathbb{Z}), & \\
U(r)=\lambda(r) \otimes 1 \otimes 1 \otimes 1 \otimes \lambda(r) \otimes 1, & r \in \chi_{p}, \\
U(g)=1 \otimes u(g) \otimes 1 \otimes 1 \otimes \lambda(g), & g \in \chi_{2} \times{ }_{s} \mathbb{F}_{2}, \\
U, & \text { corresponding to } \lambda^{\omega}(1,0, e),
\end{array}
$$

where $U$ acts on $l^{2}\left(\mathbb{Z}_{p} \times \chi_{2} \times\left(\chi_{2} \times \mathbb{F}_{2}\right) ; L^{2}\left(X_{p}, m_{p}\right) \otimes L^{2}\left(X_{2}, \mu_{\lambda}\right) \otimes l^{2}(\mathbb{Z})\right)$ via

$$
(U \xi)(n, s, h)=\left(\lambda(1) \rho_{s}^{*} \otimes 1 \otimes \lambda(1)\right) \xi(n-1, s, h) ;
$$

in each case above $\lambda(\cdot)$ denotes left translation by the appropriate element on the appropriate space, and $u(g)$ denotes the canonical unitary on $L^{2}\left(X_{2} \times Z\right.$, $\left.\mu_{\lambda} \times m_{\lambda}\right)$ arising from the action of $\chi_{2} \times{ }_{s} F_{2}$ on $X_{2} \times \mathbb{Z}$ described above.

Consider the subalgebra generated by the $\tilde{a}=a \otimes 1 \otimes 1 \otimes 1 \otimes 1 \otimes 1$ and the $U(r)$; this is nothing but the hyperfinite $\mathbb{I}_{1}$-factor $\mathcal{R}$, presented as the crossed product of $L^{\infty}\left(X_{p}, m_{p}\right)$ by $\chi_{p}$, acting by translation (see [3]). On the other hand, the subalgebra generated by $b \otimes c=1 \otimes b \otimes c \otimes 1 \otimes 1 \otimes 1$ ( $b, c$ as above) and $U(g), g \in \chi_{2} \times_{s} \mathbb{F}_{2}$ is the $\mathrm{II}_{\infty}$-factor $\mathscr{\eta}_{\lambda}$ arising in the discrete decomposition of Pukansky's factor $\mathscr{P}_{\lambda}$ (notation of [3]). This is since the action of $\chi_{2} \times{ }_{s} \mathbb{F}_{2}$ on $\left(X_{2}, \mu_{\lambda}\right)$ gives rise to $\mathscr{P}_{\lambda}$ via the group measure space construction, while the action of $\chi_{2} \times{ }_{s} \mathbb{F}_{2}$ we have described on $\left(X_{2} \times \mathbb{Z}, \mu_{\lambda} \times m_{\lambda}\right)$ is the so-called skew product of the one above, and hence gives rise to $\Re_{\lambda}$ via crossed product (see [6]). Since these two subalgebras evidently commute and the Hilbert space is already split as a tensor product, they generate $\mathscr{R} \otimes \mathscr{I}_{\lambda}$.

Furthermore, from above we easily see that

$$
U a U^{*}=\widetilde{\sigma(a)} \text { and } U U(r) U^{*}=\sigma\left(\rho_{r}\right) U(r)
$$

for $a \in L^{\infty}\left(X_{p}, m_{p}\right)$ and $r \in \chi_{p}$; thus $\operatorname{Ad} U$ on $\mathscr{R}$ gives rise to the automorphism $s_{p}^{\gamma}$ discussed in [3]. Further, by the definition of the action of $G$ on $Y$, $U U(g) U^{*}=U(g), g \in \chi_{2} \times{ }_{s} \boldsymbol{F}_{2}$, while $U(b \otimes c) U^{*}=b \otimes \theta(c)$, where $(\theta(c))(n)$ $=c(n-1)$ for $c \in l^{\infty}(Z)$. However this describes precisely the automorphism $\theta_{\lambda}$ of $\mathscr{I}_{\lambda}$ such that $\mathscr{R}\left(\mathscr{\eta}_{\lambda}, \mathscr{Z}, \theta_{\lambda}\right)$ is isomorphic with $\mathscr{Q}_{\lambda}$ (see [6]). Thus Ad $U$ on $\mathscr{R} \otimes \mathscr{N}_{\lambda}$ implements the automorphism $s_{p}^{\gamma} \otimes \theta_{\lambda}$.

Finally, if we identify the Hilbert space of the crossed product, as described above, with $L^{2}\left(X_{p} \times X_{2} \times \mathbb{Z} \times \chi_{p}\left(\chi_{2} \times{ }_{s} \mathbb{F}_{2}\right)\right) \otimes l^{2}(\mathbb{Z})$, (it is the second copy of $\mathbb{Z}$ which has been brought outside), it is clear from the formula above that $U$ 
is of the form $V \otimes \lambda(1)(\lambda(1)$ denoting translation by 1 as usual). But this means that the operators (*) generate $\mathscr{R}\left(\mathscr{R} \otimes \eta_{\lambda}, Z, s_{p}^{\gamma} \otimes \theta_{\lambda}\right)=Q_{\lambda, p, \gamma} \quad$ Q.E.D.

Remark. All other known examples of factors not anti-isomorphic with themselves (type III factors, given by Phillips [18], and examples of $\mathrm{II}_{1}$-factors, to appear in a paper by Connes, [3]) may be constructed as above i.e. by crossed product of an abelian algebra by a discrete group and a cocycle. They are, if anything, slightly more complicated than the examples $Q_{\lambda, p, \gamma}$ presented above.

In this paper, no attempt has been made at systematically computing the groups $H_{a}^{2}(G ; \mathcal{U}(\mathcal{Z}(\mathscr{M})))$; to the authors knowledge, no effective method is known for the general case. Here, however, we would like to collect brief descriptions of some of the results which are known. Throughout the discussion, $\mathcal{A}$ denotes an abelian von Neumann algebra, and $G$ a group acting as automorphisms of $\mathcal{A}$; we will also need to relaize $\mathcal{A}$ as $L^{\infty}(\Gamma, \mu)$ for some standard measure space $(\Gamma, \mu)$; in this case we let $(g, \gamma) \in G \times \Gamma \rightarrow g \gamma \in \Gamma$ be the point realization of $G$ (see [12]; [19]).

If $G$ is discrete, the whole machinery of algebraic cohomology theory is available; in particular, arbitrary projective or injective resolutions may be used to compute the cohomology (see [14]). Thus in particular $H^{n}(Z, \mathcal{Q}(\mathcal{A})$ ) $=0$ for $n \geq 2$, for any action. Suppose $G$ is freely acting on $\mathcal{A}$; then C.C. Moore and J. Feldman (in a preprint) have shown how to define the groups $H^{n}(G, \mathcal{U}(\mathcal{A}))$ using only the equivalence relation on $\Gamma$ arising from the action of $G$; thus if the action of $G$ on $(\Gamma, \mu)$ is weakly equivalent to a single transformation, $H^{n}(G, \mathcal{U}(\mathcal{A}))=0, n \geq 2$. In particular, if $G$ is abelian, finitely generated, and acts on $\Gamma$ so as to preserve a finite or $\sigma$-finite measure in the class of $\mu$, then $H^{n}(G, \mathcal{U}(\mathcal{A}))=0, n \geq 2$. The same technique shows that if $G$ acts freely and transitively on $\Gamma$ (i.e. $\Gamma=G$ ), then $H^{n}(G, \mathcal{Q}(\mathscr{A})$ ) $=0$, $n \geq 1$.

If $G$ is not discrete, less is known; the techniques of spectral sequences are available (as in the discrete case), but only in a somewhat limited form (see [15]). However, if the action of $G$ on $\Gamma$ is the "closure of the range" of a homomorphism from the action of a discrete group $H$ on $(X, \nu)$ (see [13]), then $H^{n}\left(H, \mathcal{U}\left(L^{\infty}(X, \nu)\right)\right)=H^{n}(G, \mathcal{U}(\mathcal{A}))$ for all $n \geq 0$. The proof of this fact follows exactly the proof given in [6] in the case $H=\boldsymbol{Z}, G=\boldsymbol{R}$. In particular, by Ambrose's Theorem, [13], $H^{n}(\boldsymbol{R}, \mathcal{U}(\mathcal{A}))=0$ for $n \geq 2$, for any action of $\boldsymbol{R}$ on $\mathcal{A}$ which is ergodic. 


\section{References}

[1] Connes, A., Une classification des facteurs de type III, Ann. de l'Ecole Normale Superiéure, 6 (1973), 133-252.

[2] - Almost periodic states and factors of type $\mathrm{III}_{1}$, J. Funct. Analysis, 15 (1974), 217-243.

[3] —, A factor not anti-isomorphic to itself, Ann. Math.,101 (1975), 536-553.

[4] - Outer conjugacy classes of automorphisms of factors, Ann. Sci. École Norm. Sup. (4), 8 (1975), 383-419.

[5] — Classification of injective factors, Ann. Math., 104 (1976), 73-116.

[6] Connes, A. and Takesaki, M., The flow of weights on a factor of type III, Tôhoku Math. J., 29 (1977), 473-575.

[7] Digernes, T., Duality for weights on covariant systems and its applications, thesis, UCLA, 1975.

[8] Eilenberg, S. and Maclane, S., Cohomology theory of abstract groups I, Annals of Math., 48 (1947), 51-78.

[9] Haagerup, U., The standard form of von Neumann algebras, Math. Scand., 37 (1975), 271-283.

[10] Halpern, H., One parameter automorphism groups of generalized $\mathrm{K}-\mathrm{H}$-algebras, Preprint, U. of Cincinnati.

[11] Macduff, D., Central sequences and the hyperfinite factor; Proc. London Math. Soc., XXI, (1970), 443-461.

[12] Mackey, G., Point realization of transformation groups, Illinois J. Math., 6 (1962), 327335 .

[13] — Ergodic Theory and Virtual groups, Math. Ann., 166 (1966), 187-207.

[14] Maclane, S., Homology, Academic Press, New York, 1963.

[15] Moore, C. C., Group extensions and cohomology for locally compact groups III, Trans. Amer. Math. Soc., 221 (1768), 1-34.

[16] Nakamura, M. and Takeda, Z., On the extensions of finite factors, Proc. Japan Academy, 35 (1959), 149-154.

[17] Paschke, W., Inner product modules over $B^{*}$-algebras, Trans. Amer. Math. Soc. 182 (1973), 443-468.

[18] Phillips, J., Automorphisms of full $\mathrm{II}_{1}$-factors, with applications to factors of type III, Duke Math. J., 43 (1976), 375-385.

[19] Ramsay, A., Virtual groups and group actions, Advances in Mathematics, 6 (1971), 253322.

[20] Rieffel, M., Induced representations of $C^{*}$-algebras, Advances in Math., $\mathbb{1 3}$ (1974), 176-257.

[21] Sutherland, C., Cohomology and extensions of von Neumann algebras II, Publ. RIMS, Kyoto Univ., 16 (1980), 135-174.

[22] Takesaki, M., Tomita's theory and its applications, Lecture Notes in Math., 128, Springer, 1970.

[23] — Duality for crossed products and the structure of von Neumann algebras of type III, Acta Math., 131 (1973), 249-310.

[24] Zeller-Meier, G., Produits croisés d'une $C^{*}$-algebra par un groupe d'automorphisms, J. Math. Pures et Appliques, 47 (1968), 101-239. 
